\title{
Construcción de una metodalogía para evaluar la sustentabilidad de las políticas de inversión en innovación
}

\section{Tatiana Giraldo Pardo*}

Methodalogy design to

evaluate the

sustainability of

investment policies for

innovation

Construction d'une

méthodalogie

d'évaluation de la

durabilité des

politiques

d'investissement en

innovation

Canstruçãa de uma

metodologia para

avaliar a sustentabili-

dade das políticas de

*Administradora de Empresas, Universidad Nacional de Colombia.

Magister en Ingenieria Industrial, Universidad de los Andes. D.U. en Sciences de Gestion, Universite de Rouen (Francia). Estudiante del Doctorado en Direccion de Empresas de la Universidad Pablo de Olavide (Sevilla-Espana). Actualmente Coordinadora de Investigacion y Desarrollo de la Facultad de Administracion de Empresas de la Universidad Externado de Colombiay investimento em docente de Métodos Cuantitativos aplicados a la Gestión. in๊vaçã⿰ 


\section{RESUMEN}

Este trabajo pretende identificar a la luz de los indicadores de ciencia y tecnología, cuáles factores determinan la sustentabilidad de las políticas de inversión en Ciencia, Tecnología e Innovación dentro de los países iberoamericanos. Para ello se adoptan las cifras de los indicadores de la Ricyt (Red Iberoamericana de Ciencia, Tecnología e Innovación), y con base en los resultados, se construyen dos modelos de sustentabilidad, fundamentados en dos ecuaciones paralelas. El primer modelo, está en función de la sostenibilidad y la pertinencia de las actividades de Ciencia y Tecnología. El segundo modelo, hace depender la sustentabilidad de la responsabilidad social y la iniciativa a la estructura. Se espera que los resultados arrojados por este doble modelo, sean más robustos que aquellos formulados a partir de los resultados de cada uno de los métodos por separado..

\section{ABSTRACT}

This study aims at identifying the indicators in the fields of science and technology, which factors determine the sustainability of investment policies on science, research and innovation, among Iberoamerican countries. For this, Ricyt indicators have been adopted (Iberoamerican Network of Science, Technology and Innovation), and based on results, two sustainability models are designed focused on two parallel equations. The first model supports sustainability and appropriateness of scientific and technological activities. The second model, however, describes the dependence on sustainability based on social responsibility and structure initiative. It is really expected that the results shown by these two models be more robust than those resulting from each of these models in isolation.

\section{RESUMÉÉ}

Ce document vise à identifier, grâce à certains indicateurs scientifiques et technologiques, les facteurs déterminant la viabilité des politiques d'investissement en Science, Technologie et Innovation dans les pays d'Amérique latine. Pour ce faire, les chiffres et indicateurs de la Ricyt (Réseau ibéro-américain de la science, la technologie et l'innovation) sont pris en compte, et selon les résultats obtenus, deux modèles de développement durable sont construits, basés sur deux équations parallèles. Le premier modèle repose sur la viabilité et la pertinence des activités scientifiques et technologiques. Le second modèle fait dépendre la structure de la durabilité à la responsabilité sociale des entreprises et à l'initiative. Les résultats attendus de ce double modèle devraient être plus robustes que ceux réaliser séparément à partir des résultats de chacune des méthodes.

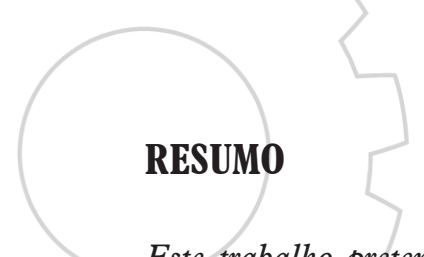

Este trabalho pretende identificar à luz dos indicadores de ciência e tecnologia, quais factores determinam a sustentabilidade das políticas de investimento em Ciência, Tecnologia e Inovação nos países iberoamericanos. Para isto adotaram-se os valores dos indicadores da Ricyt (Rede Iberoamericana de Ciência, Tecnologia e Inovação), e com base nos resultados, construem-se dois modelos de sustentabilidade, fundamentados em duas equaciones paralelas. O primeiro modelo está em função da sustentabilidade e a relevância das actividades de Ciência e Tecnologia. O segundo modelo, faz depender a sustentabilidade da responsabilidade social e a iniciativa da estrutura. Espera-se que os resultados obtidos por este duplo modelo sejam mais robustos do que aqueles formulados a partir dos resultados de cada um dos métodos de forma separada.

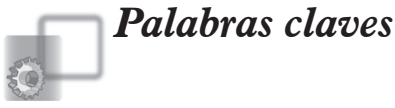

Politicas de inversión en Ciencia

Tecnología e Innovación

Sustentabilidad

Pertinencia

Responsabilidad Social

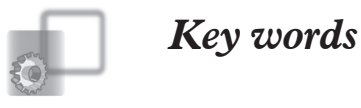

Investment policies on science Technology and innovation Sustainability a Appropriateness Social responsibility

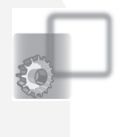

\section{Mots clefs}

Investissements scientifiques Technologie et innovation

Durabilité

Pertinence

Responsabilité sociale des entreprises.

\section{Palavras-chave}

Políticas de investimento em ciência

Tecnologia e inovação

Sustentabilidade

Pertinência

Responsabilidade social 


\section{INTRODUCCIÓN}

E los últimos años, se ha venido dando un desplazamiento del interés académico de los temas que respectan solamente a la factibilidad económica de los proyectos, como criterio de desempeño, hacia el uso de otros términos más complejos tales como la responsabilidad social, la sostenibilidad y posteriormente la sustentabilidad.

Este trabajo pretende hacer una consideración más amplia de las variables que determinan el desempeño las actividades de inversión en innovación, con miras a establecer una metodología que sirva para su evaluación, esto en términos de la sustentabilidad de dichas actividades, lo cual nos permitirá hacer clasificaciones por países en términos de la responsabilidad social, la sostenibilidad y finalmente la sustentabilidad inherente a sus políticas y actividades de fomento a la ciencia, la tecnología y la innovación.

Sin embargo, pese a la proliferación de literatura en los temas de innovación, por un lado, y sustentabilidad por el otro, el primer aporte de este trabajo será establecer una sinergia entre esos dos grandes temas, teniendo en cuenta que hasta el momento, son muy escasos los trabajos que lo han intentado.

No obstante, como ya se mencionó, la importancia de este trabajo radica en la necesidad de toma de decisiones en materia de política pública, y por consiguiente de los entes que integran los Sistemas Nacionales de Innovación de los distintos países iberoamericanos.

Así, el punto de partida para establecer la importancia de la toma de decisiones sobre la inversión en innovación, se encuentra que este, no es sólo un problema económico, sino que a su vez, incorpora un contenido difuso de cara a la evolución y crecimiento de las instituciones. Así, desde los planteamientos de Bowles (2005), la innovación no puede ser atrapada en indicadores lineales, para ello, se requieren expresiones matemáticas que reflejen la dimensión holística- cultural del proceso, que en su expresión de control pueda ser asimilada a raíz de una decisión, la cual puede expresarse a partir de criterios dinámicos.
Para comenzar, es posible encontrar un sistema que establezca el problema de inversión en innovación a través de los escenarios de productividad, competitividad y sustentabilidad. Bajo este marco analítico, se enfatiza que la información y el conocimiento son los ejes fundamentales de la función de producción de las instituciones que llevan a cabo actividades de investigación y desarrollo, la cual está representada en su mayor porcentaje en el capital humano y en segundo lugar, en la tecnología.

Desde esta perspectiva de la competitividad, el conocimiento es el principal factor, pero no de orden lineal, sino de orden complejo superior y cada avance significativo implicará redefiniciones trascendentes en muchos espacios de la estructura productiva de lo urbano, de los sectores productivos y la sociedad misma. Con ello, se quiere expresar que el eje fundamental de la competitividad, se encuentra quizá en el capital humano haciendo de la misma un proyecto sostenible (Henderson y Cockburn, 1994) en el sentido de la innovación permanente (Schumpeter, 1950).

Ahora bien, a partir de las definiciones los espacios sociales en los cuales, la relación fundamental de productividad es la derivada de la intercepción de las capacidades de los individuos, y no de estos en su potencialidad de cambio de los bienes de origen primario propios de la economía industrial, es que puede pensarse la innovación como probabilidad concretada en el rango y dominio social. Es decir, que innovación, como cultura, la hay a lo largo de toda la sociedad por la innata curiosidad de los individuos. El problema es, si los mecanismos de la sociedad (institucionales, de mercado y de no-mercado) posibilitan y potencian su construcción o antes bien la detienen. Esta es la hipótesis central: que esto no produce innovación de manera significativa y que sus mecanismos institucionales tampoco coadyuvan de manera trascendental en las ciudades y contribuyen a justificar su estado de desarrollo.

Por lo tanto, se concluye que, una de los principales determinantes del proceso innovador, se ve reflejado en que las organizaciones, de acuerdo con Nonaka, Toyama 
y Nagata (2000), deben crear sus propias ventajas competitivas en repuesta a las necesidades del mercado, donde la capacidad de crear y utilizar conocimientos es el origen más importante de estas. Así, los conocimientos y las destrezas de los agentes que participan en el proceso de innovación dan una ventaja competitiva a una organización porque es a través de este juego de conocimientos y destrezas que ella puede innovar en sus productos, procesos y servicios, o mejorar los ya existentes eficiente y eficazmente.

Por otra parte, estudiar el proceso de la inversión en innovación como fomento, sólo puede entenderse si se sitúa dentro del medio ambiente en el que se desarrolla, por lo tanto, es vital analizar el ecosistema de la innovación, que incluye los inputs o fuentes de la innovación; los outputs y sus correspondientes efectos sobre la sociedad en su conjunto y los distintos agentes que la conforman; los condicionantes políticos y la infraestructura sobre las que se asienta ese proceso de inversión. De este modo, los incentivos de inversión en innovación del sector servicios están atados a la competencia interina de las firmas por mantener los canales de mercado ya establecidos y la necesidad de mantener relaciones de complementariedad con sectores como el manufacturero.

Este trabajo tiene como objetivo identificar los factores que determinan la sustentabilidad de las actividades de inversión en innovación, y a partir de ellos construir una metodología que permita evaluar la sustentabilidad de dichas actividades, lo cual se convierte en un insumo importante para las decisiones institucionales y de política pública. En este sentido, se espera que los resultados proporcionen elementos para la construcción de una política pública con miras a forjar mayores niveles de innovación en los países.

En el desarrollo de este trabajo, se pretende confrontar aspectos sistémicos y analíticos (inferencial), por cual podría decirse que es de naturaleza constructivista, ya que tiene como marco de referencia dos paradigmas epistemológicos distintos. Sin embargo, en esta ocasión el aspecto sistémico solamente se utilizará como mapa mental para el desarrollo de la investigación, en la cual se hará énfasis en los aspectos analíticos.

No obstante la lógica del paradigma lineal, aunque aparentemente sencilla, la situación es más compleja cuando se intenta hacer una inversión en innovación en un contexto tan particular como el educativo, porque en el proceso de toma de decisiones que se lleva a cabo en la realidad, no sólo se tiene en cuenta el riesgo o el rendimiento, necesariamente. También son relevantes las capacidades técnicas y operativas de la organización, así como otras variables del ambiente externo ¿Cómo tomar una decisión de inversión acertada, evitando el riesgo de perder la oportunidad de inversión?

Al hacer una revisión de los modelos econométricos, se encuentra implícito el paradigma cartesiano, que constituye el soporte ontológico del reduccionismo y que conduce a que la econometría realice representaciones fragmentadas de la realidad en detrimento de la capacidad explicativa de los modelos. Al usar los modelos econométricos, el tomador de decisiones está dejando fuera de consideración, aspectos que podrían ser relevantes a la hora de elegir entre varias opciones.

Por otro lado, el pensamiento sistémico como método de construcción de conocimiento "es impulsado continuamente por un afán holista" (Andrade, Dyner, Espinosa, López y Sotaquirá, 2003) y a través del reconocimiento de diferentes perspectivas de un fenómeno, hace cada vez más completo el conocimiento sobre dicho fenómeno. A partir del paradigma sistémico, se construye conocimiento en el marco de una visión global e integral de los fenómenos; denotando los cambios observados en los fenómenos como el resultado de la dinámica de las múltiples relaciones de influencia entre los elementos que conforman el sistema que representa al fenómeno objeto de estudio.

Este nuevo paradigma, cumple con los criterios Kuhnianos para validar un nuevo paradigma que intenta reemplazar a otro más viejo en un campo específico a saber:

- Que la teoría sea precisa en sus predicciones para que haya una mayor conexión entre realidad y teoría.

- La consistencia teórica.

- El alcance amplio y la simplicidad en la teoría.

Al parecer, todos estos criterios son satisfechos en un grado elevado por la teoría y la dinámica de sistemas, si bien, su principal objetivo es el de hacer una representación 
de la realidad que tenga en cuenta las interacciones entre sus diferentes componentes o elementos, para dar un descripción más exacta de los fenómenos que intenta representar.

Kuhn, señala que finalmente la acumulación de anormalidades hace cambiar a un paradigma observaciones que no tienen cabida y que no pueden ser explicadas por los paradigmas actuales. Estas anomalías tienen que ver en el caso de los modelos econométricos, con el excesivo grado de reduccionismo implícito en su formulación. Los modelos lineales para evaluar la viabilidad económica de los modelos de inversión, se han desarrollado basándose en fuertes supuestos así como en la correlación de los rendimientos de los activos. En ellos sólo se consideran los dos primeros momentos de la distribución de los retornos: la media y la varianza. Esta simplificación no supone inconvenientes cuando los retornos de los activos presentan distribución normal, pero infortunadamente esto en la práctica no ocurre.

En cuanto a la parte analítica, se hará uso de los Framework Spaces de Harrod, complementándolo con un análisis gráfico que parte de las variables involucradas en algunas de las definiciones más usadas y relevantes del término sustentable, las cuales se desarrollarán a continuación en el marco teórico. Para llevar a cabo este ejercicio, como terreno de observación, se tiene el conjunto de países iberoamericanos e interamericanos, en términos de sus indicadores de ciencia y tecnología, los cuales serán tomados de la RICYT (Red de Indicadores de Ciencia y Tecnología), para los años 1997, 2002 y 2007.

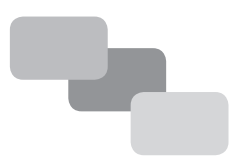

\section{Z. IDENTIFILACIÓN DEL PRDBLEMA}

\subsection{Marco teórico y estado del arte}

\subsubsection{Sustentabilidad, sostenibilidad y respon- sabilidad social}

De acuerdo con Lopez-Ricalde (2005), la definición de desarrollo sustentable se ha ido ajustando gradualmente al incrementarsen condiciones sociales en donde el ser humano es parte de un sistema y no dueño del mismo. En primer lugar, la sustentabilidad se entiende como la capacidad de un proyecto para continuar siendo viable a largo plazo. Por tanto, está directamente relacionada con la capacidad organizacional de conducirla, es decir, con la sostenibilidad (Letelier, 2002) y con la pertinencia, definida como la utilidad pública una actividad, en este caso económica.

Por una parte, las condiciones de sostenibilidad, están asociadas con variables como la viabilidad financiera y la estabilidad presupuestaria, que permiten la ejecución eficaz y eficiente de los procesos de una empresa u organización (Letelier, 2002).

El financiamiento, por lo tanto, debe cubrir los gastos necesarios, para que las actividades productivas respondan a las demandas del entorno y a las expectativas de población. También son necesarios recursos como infraestructura física, la tecnología y los servicios de información. Además, debe demostrarse no sólo que estas actividades o proyectos dispondrán de recursos, sino también que las fuentes de esos recursos se mantendrán o variarán positivamente en el tiempo.

La segunda condición de la sostenibilidad, tiene que ver con la gestión de los recursos humanos estableciéndose las competencias que se requieren para conformar cada equipo, así como los plazos y las estrategias para alcanzar los propósitos propuestos (Letelier, 2002). Por lo tanto, las condiciones de sostenibilidad, no sólo están relacionadas con la eficiencia en el uso de los recursos, sino también con temas éticos. 
Por otra parte, la sustentabilidad se relaciona también con la capacidad organizacional de adaptarse a las condiciones cambiantes del entorno (político, económico, cultural, educacional, demográfico, etc).
Aunque la sustentabilidad de las empresas depende de la pertinencia social de estas y de la capacidad interna de sostenerlas con una gestión efectiva, hay formas alternativas de concebir la sustentabilidad (Ver figura 1).

Figura 1. Sustentabilidad, sostenibilidad y responsabilidad social: Conceptos

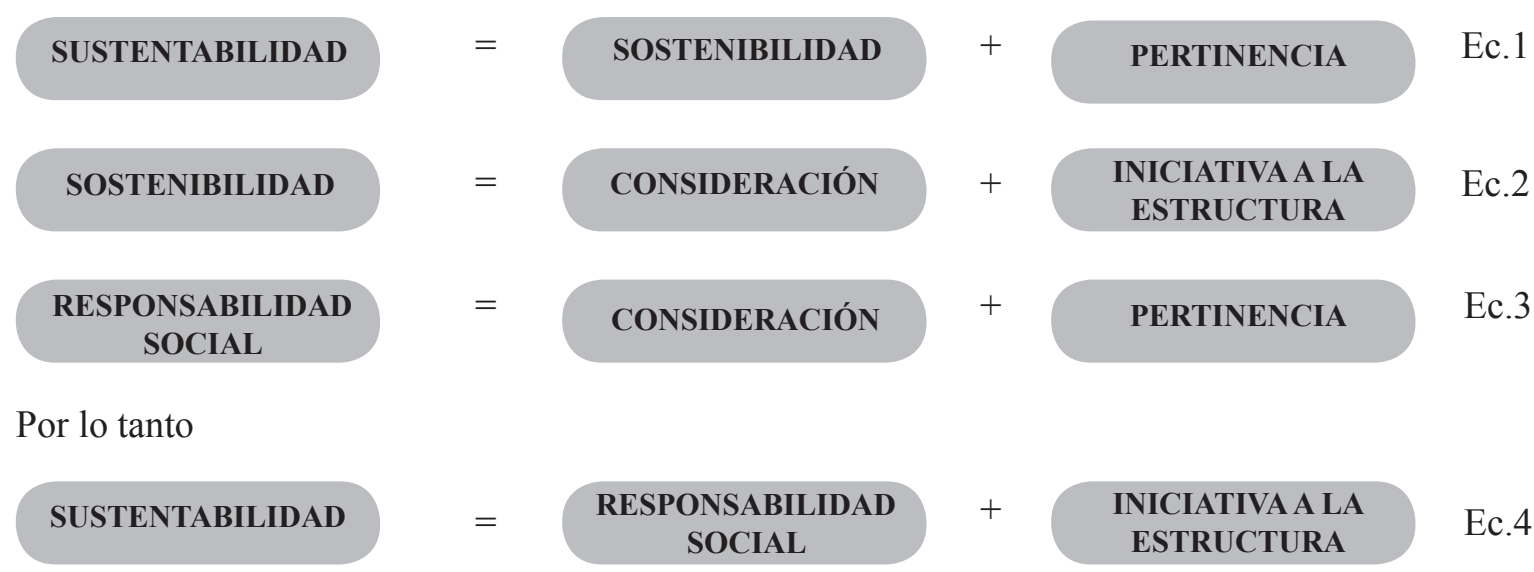

Fuente. Elaboración propia.

En la ecuación dos, se concibe la sostenibilidad como el resultado del ejercicio de la gestión, en los términos en que la define Robbins (2004), teniendo en cuenta las dimensiones de consideración con el recurso humano e iniciativa para la estructura.

De acuerdo con la cuarta ecuación del diagrama, la sustentabilidad es el resultado de una orientación a la estructura, en términos principalmente de la orientación a los objetivos y metas económicos de la empresa, y la responsabilidad social, que no es más que la combinación entre la consideración al recurso humano, teniendo en cuenta claro está, el componente ético, y la pertinencia social y ambiental de las acciones de la empresa.

\subsubsection{El carácter dual de la innovación}

Por otro lado, es importante recordar que toda innovación tiene un carácter dual. De acuerdo con el modelo dualcore (Daft, 1978), dentro de la organización (o sistema en este caso), coexisten dos áreas diferenciadas donde puede surgir la innovación, la técnica y la administrativa, aunque cada una se caracteriza por tener objetivos, actividades y participantes diferentes. Según la perspectiva del sistema sociotécnico, el buen funcionamiento de la organización requiere que los dos sistemas se encuentren en equilibrio. Una empresa no debe introducir innovaciones de un tipo, si no adopta también cambios en el otro sistema, ya que este desequilibrio redundaría en un menor desempeño de ambos. Esta noción, atiende tanto a los distintos procesos de generación y adopción de la innovación, como a la propia naturaleza de la innovación y de la organización, en la que se pueden encontrar dos áreas diferenciadas. Podemos deducir entonces, que existirán variables (o actores, si se habla de Sistema de Innovación) que estén más directamente relacionadas con un tipo de innovación $\mathrm{u}$ otro, o por lo menos, que faciliten o dificulten un determinado proceso de adopción de la innovación.

La teoría de la estructuración de Giddens (1998), se ha movilizado para interpretar situaciones de dualidad en las organizaciones y sistemas. De hecho, la resolución de la dicotomía entre la estructura (organizacional) y la acción (innovación, en este caso), es la motivación para la teoría de la estructuración, que se basa en la idea de que la estructura, considerada como una característica inesperada de las interacciones sociales, es la entrada y la salida de acciones humanas (Giddens, 1998). Este marco se ha utilizado por ejemplo para describir la interacción entre 
las tecnologías de información avanzadas y las estructuras sociales (Chanal, 2002). En el contexto del diseño y de la innovación organizacional, resulta particularmente relevante interpretar el dilema acción/ estructura y los dilemas de la explotación/ exploración (Chanal, 2002). La estructura de la organización (o el sistema) se puede considerar entonces como un recurso y una restricción a las prácticas de innovación, es decir, los conceptos de organización e innovación están ligados de una manera recurrente o estructural (Chanal, 2002).

Figura 2. Carácter dual de la innovación

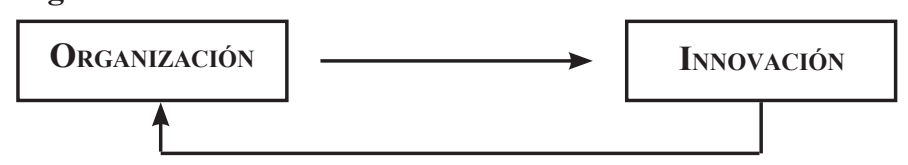

Fuente. Chanal, 2002

Esta idea de la naturaleza recurrente de la innovación, puede ser extendida a todos los niveles que conforman el Sistema Nacional de Innovación. Por lo tanto, es de esperarse que en la medida en que se hacen innovaciones en uno de los niveles, por ejemplo el micro, el sistema de innovación entendido como un conjunto de actores interactuantes, tienda a cambiar en estructura o composición.

\subsubsection{La sustentabilidad en el ámbito de la inno- vación: La sostenibilidad, y la pertinencia}

Teniendo en cuenta la ecuación 1 de la figura 1, para evaluar la sustentabilidad de un proyecto de inversión en innovación en las IES, se necesitará evaluar dos aspectos:

- La sostenibilidad del proyecto entendida como aquella característica que coadyuva a su viabilidad financiera y a su estabilidad presupuestaria a corto, mediano y largo plazo, permitiendo la ejecución eficaz y eficiente de los procesos de una empresa u organización (Letelier, 2002). De esta forma, se entiende por desarrollo sostenible aquel tipo de desarrollo que satisface las necesidades del presente sin comprometer de las generaciones futuras de satisfacer las propias. En ese sentido, este tipo de desarrollo propende por que los sistemas y las instituciones de ciencia y tecnología, creen capacidades internas a través del aprendizaje, que puedan ser apropiadas por sucesivas generaciones futuras de agentes innovadores en un proceso que podría catalogarse como recursivo.
- La pertinencia del proyecto, está asociada con el dar respuesta a la pregunta ¿A quién le interesa el desarrollo de este proyecto? Por consiguiente, se busca que los proyectos se formen con cierto grado de adecuación a las condiciones de la sociedad, el mercado, la competencia, el estado del arte de la tecnología, los recursos y oportunidades para desarrollar un proyecto.

La pertinencia de un proyecto se considera también con relación a los objetivos, intereses y motivaciones de los diversos agentes para llevar a cabo el desarrollo de un proyecto. También puede verse como la justificación del proyecto en relación con las prioridades de desarrollo (Crespo, M.,2009).

Sobre el primer aspecto, la sostenibilidad, en Schumpeter la innovación se convierte en la fuente de la dinámica capitalista y en su explicación funge como un fenómeno netamente endógeno. De esta forma, el crecimiento y el ciclo económico se funden en un solo concepto. La dependencia del progreso tecnológico de factores económicos tales como las condiciones de demanda, gastos de investigación y desarrollo y efectos

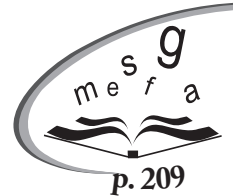
de learning by doing, implica que cambios en el lado de la oferta no son independientes de los que ocurren en el lado de la demanda. Por consiguiente, la sostenibilidad de la innovación a través del tiempo, supone un esfuerzo en términos de aprendizaje organizacional, teniendo en cuenta que este es necesario para adaptar las innovaciones a la estructura de la organizaciones productivas, y a su vez, que las innovaciones posteriores que se generen dentro de la innovación sean efectivas y permitan generar ciclos sucesivos de mejora continua. Por tal motivo, la sostenibilidad de una inversión, puede verse como un resultado directo de los ciclos de aprendizaje organizacional o institucional.

De esta forma, Argyris y Schon, sostienen que básicamente las organizaciones que desarrollan el aprendizaje organizacional inician con simples procesos antirutinarios, que no cuestionan la estructura de la organización, sus interrelaciones con el entorno, sus valores o sus procesos de toma de decisiones. Luego se adentra en un segundo nivel en el que se busca la reestructuración organizacional, partiendo desde el aprendizaje individual, y que cuestiona la racionalidad detrás de las acciones. 
Teniendo en cuenta que la inversión es una variable de tipo económico, el desarrollo económico es sostenible

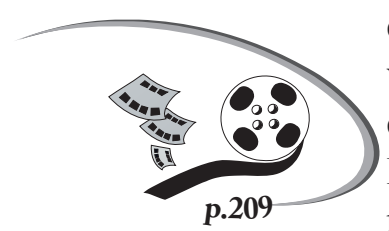
cuando se puede sostener una determinada variable crucial, sin disminuir en el futuro como consecuencia del propio crecimiento. En este sentido, se denominará como sostenibilidad económica, al crecimiento que está basado en el crecimiento del producto; y sostenibilidad social como aquel crecimiento acompañado paralelamente del aumento del empleo; todo ello amparado en el crecimiento de la industria (Kaldor, 1975).

No obstante, en la bibliografía sobre la materia, los enfoques del desarrollo sostenible pueden clasificarse en tres grupos: si la variable crucial o la función objetivo a maximizar es el bienestar (o la utilidad), el consumo, o el capital (manufacturado o natural). La elección de la variable crucial tiene importantes implicaciones, puesto que su sostenibilidad a menudo entraña la insostenibilidad de otros candidatos posibles a cumplir esta función (Vercelli, 1998). Este autor propuso que la variable básica que debe conservarse a través del tiempo mediante el desarrollo económico sería la libertad para las generaciones futuras.

En relación con el segundo aspecto, en el ámbito de la ciencia y la tecnología, la pertinencia se puede dividir en dos grandes ámbitos: pertinencia educativa y pertinencia social. La pertinencia, está definida por autores como Latapí (1995), citado por Tünnermann (2006), como la dimensión que comprueba que los objetivos propuestos por la institución corresponden a los requeridos desde una perspectiva externa. Esta definición de pertinencia científica da pié al nacimiento de otras conceptualizaciones acordes con la naturaleza de esta perspectiva con la que estén relacionados los objetivos educativos. En ese sentido, se habla de pertinencia social, política, económica, cultural y hasta pedagógica, dependiendo de si los fines investigativos se vinculan o no con las necesidades $y$ demandas de una determinada sociedad, ideología política, sector productivo, cultura o institución. Por ello, Malagón (2003) propone el concepto de pertinencia integral en la que el currículo se presenta como un eje central de la relación existente entre la escuela y los factores socioeconómicos, políticos y culturales de su entorno. Tünnermann (2006) afirma que "la valoración de la pertinencia no es una tarea fácil especialmente si se adopta el concepto amplio de pertinencia social. La pertinencia exige flexibilidad curricular y desplaza el énfasis en la transmisión del conocimiento, de los procesos de enseñanza a los de aprendizaje, centrándolos en el estudiante", quien es el que aprende y tiene que construir el conocimiento e incorporarlo a su estructura cognitiva.

Para los organismos multilaterales y para las autoridades educativas, la pertinencia se reduce al vínculo productivo. Esta visión utilitarista ha alcanzado incluso a los diversos stackeholders (principalmente las empresas) quienes valoran la investigación en función de su potencial práctico. El sesgo utilitarista tiende a jerarquizar los conocimientos, y por lo tanto los aprendizajes, en dos tipos básicos: los que construyen capacidades y destrezas aplicables, y el resto. Este enfoque tiene otras repercusiones: se manifiesta en esquemas de investigación y desarrollo basado en competencias.

En este sentido, Cajiao (2008) establece la diferencia que hay en el concepto de pertinencia, entendido desde la óptica funcional de la $\mathrm{I}+\mathrm{D}$ con respecto al sistema productivo y desde la percepción y necesidades específicas de los ciudadanos que acceden a las instituciones educativas.

En la primera perspectiva -que suele ser la más frecuente en la literatura-, la pertinencia se relaciona con las necesidades de la sociedad, especialmente en su aspecto productivo, pretendiendo adecuar lo que se ofrece desde la educación formal a la demanda potencial del mercado laboral. De allí provienen muchas orientaciones de política pública relacionadas con el diseño de programas académicos de corte técnico y tecnológico, así como la orientación que debería darse a ciertas áreas del conocimiento como la matemática, el aprendizaje del inglés o el énfasis en las llamadas competencias laborales. En ese sentido, la pertinencia social se define como la relación de la ciencia y la tecnología con la sociedad y obliga a las instituciones y centros de investigación a responder sobre lo que estos generen como productos y servicios en relación con las expectativas sociales. Permite la interlocución efectiva de las instituciones de CyT con los actores de su medio, ofreciendo respuestas concretas, apropiadas y oportunas a sus necesidades. La pertinencia social significa la capacidad creativa que tiene la investigación para responder en forma proactiva a las necesidades de su entorno. 
Por consiguiente, es preciso que los investigadores asuman como objeto de estudio de sus programas y proyectos, los problemas de la realidad local, regional y nacional, comprometiéndose de esa manera a la búsqueda de soluciones y a la generación de alternativas para el desarrollo productivo y social; dichos planteamientos y soluciones se convertirán a su vez en conocimientos que al ser sistematizados, contribuirán como materia prima para la readecuación de los planes y programas de formación de los investigadores, lo cual implica nuevamente un proceso de aprendizaje (Arellano, 2003).

\subsubsection{La sustentabilidad en el ámbito de la innovación: responsabilidad social e iniciativa a la estructura}

\subsubsection{Responsabilidad social}

El diseño de las políticas públicas de innovación, ha cobrado vital importancia en el sentido de que recoge una serie de cambios estructurales en la economía nacional, que afectarán de diversa manera a las instituciones de educación superior, en las distintas regiones del país. Aquí cobra especial interés la formación de redes de innovación en dichas instituciones, lo cual está directamente asociado con la construcción y formación de capital social en torno a las IES y sus prácticas de innovación.

Los diversos factores pertenecientes a los distintos niveles del modelo nacional de competitividad (micro, meso, macro y meta), consolidan y complementan la construcción de un entorno favorable a la innovación,

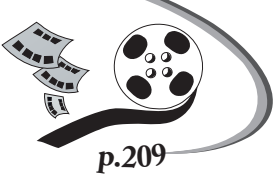
a la comunicación y a la calificación necesarias para insertarse en la competitividad internacional. De ahí la necesidad de impulsar la colaboración entre actores (individuos e instituciones) de ámbitos locales para crear redes de innovadores, que mediante su interacción y coordinación creen mecanismos de sinergia y economías de escala que propicien un entorno favorable al desarrollo industrial.

Así, para el enfoque evolucionista, en la innovación existe un alto grado de inseguridad por una creciente densidad del conocimiento, por la formalización de investigación y desarrollo (IyD) a nivel de empresas y por el papel complementario de los procesos informales de aprendizaje y los efectos acumulativos. En el proceso de innovación de las organizaciones, estos factores dependen de la interacción dinámica de las competencias; las mismas son esenciales porque enfatizan el conocimiento tácito y los procesos de aprendizaje informal, cuya especificidad depende de las tecnologías y la cultura organizativa empresarial.

La información, los conocimientos y las habilidades surgen de la acumulación local de know-how específico y del conocimiento formal, generados en procesos formales e informales de aprendizaje que se desarrollan entre las empresas y las organizaciones que conforman el marco institucional, nacional y regional de una IES. Esto constituye a lo largo del tiempo un patrimonio de competencias que determinan la capacidad innovativa de la organización.

Así como ya se ha mencionado, dentro del proceso de innovación sobresalen factores complementarios como la calificación (de los recursos humanos) y la organización (la inserción de la tecnología en los contextos sociales y procesos de producción). De ahí que la tecnología no puede separarse del entorno en el que surge ni de las estructuras organizativas donde se utiliza. El capital social de una IES, estará entonces conformado por el conjunto de asociaciones o redes, las cuales constituyen los vínculos que ligan los conjuntos de actores diferenciados a través de relaciones implícitas o explícitas que van desde el simple conocimiento hasta la cooperación.

\subsubsection{Iniciativa a la estructura}

De acuerdo con Winter (2000), la acumulación de capacidades es una condición para el logro de determinados objetivos, que tienen que ver con el desempeño futuro de la puesta en marcha de una tecnología, en este caso al interior de los procesos de enseñanza-aprendizaje. Las capacidades organizacionales están asociadas con las posibilidades de las organizaciones

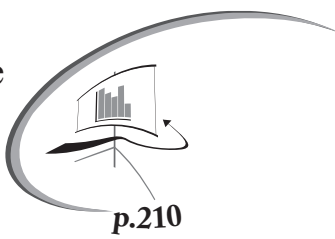
para abordar de manera satisfactoria la solución de los problemas que surgen en el proceso de introducción y utilización de una nueva tecnología. En este sentido, la existencia de capacidades asegura la superación de los problemas que emergen en el proceso, lo que da como resultado la incorporación exitosa de la tecnología. 
Para abordar este tema, se adopta la aproximación sugerida por Bell (1984), en el sentido de diferenciar las capacidades operativas -aquellas que permiten mantener operativa una tecnología- de las capacidades tecnológicas -aquellas que permiten dominar y dirigir el cambio técnico-. En consecuencia, es posible identificar distintos escenarios evolutivos del proceso de introducción de una nueva tecnología, según el nivel de capacidades que las organizaciones logren acumular en función de los distintos procesos de aprendizaje tecnológico puestos en marcha, como se verá más adelante.
Como resultado de la aplicación de las capacidades acumuladas se obtiene, principalmente, un aumento en la eficiencia operativa o tecnológica de institución y por ende, una mayor rentabilidad a mediano y largo plazo, lo que se puede asociar con procesos de aprendizaje de circuito simple (en caso de que se trate de aprendizaje meramente operativo) o de circuito doble (en caso de que se trate de aprendizaje tecnológico, en el sentido señalado por Freeman y Pérez (1988).

\section{RESULTADUS}

\subsection{Modelo de dinámica de sistemas}

Para profundizar en la comprensión de las relaciones entre las variables que se asocian con las decisiones de inversión, ciencia y tecnología, se propone inicialmente un modelo causal de dinámica de sistemas que se sustenta de la siguiente forma:

- Las capacidades organizacionales (operativas y tecnológicas) se acumulan como resultado de procesos de aprendizaje, que a su vez son posibilitados por los capitales social y humano de la comunidad. El componente social de este capital aporta las redes y conexiones, las organizaciones formales, las relaciones de confianza, la reciprocidad y el intercambio, que convierten a la comunidad en un sistema social en el cual sus agentes están en interacción mutua y con entidades externas. Entre tanto, el componente humano, la base de aptitudes, conocimientos y competencias laborales de los individuos.

Por lo tanto, los capitales social y humano que las instituciones de ciencia y tecnología requieren para sostener procesos de aprendizaje, son el resultado de una adecuada inversión social gubernamental y de la eficiencia de la comunidad en transformar lo que produce aptitudes, conocimientos y competencias laborales individuales, redes, conexiones, organizaciones y relaciones de confianza, reciprocidad e intercambio, adecuadas para una exitosa introducción de las tecnologías en cuestión. A su vez, una institución con suficiente capital social estará en mejor posición de interactuar con el gobierno central y con otras entidades para lograr un mayor apoyo (en términos de inversión social, por ejemplo).

- Dependiendo de las tecnologías involucradas en el proceso de innovación, estas presentarán grados determinados de dificultad operativa y de dificultad tecnológica. La dificultad operativa está asociada con la frecuencia y la gravedad de los problemas operativos que de la puesta en marcha de las tecnologías. El surgimiento de tales problemas es fundamentalmente aleatorio y su solución, a medida que aparezcan, garantizará la operación estable del proceso productivo, ya que se logrará un buen desempeño de la tecnología $\mathrm{y}$, por lo tanto, niveles adecuados de potencia eléctrica, disponible para la comunidad.

Por otra parte, la dificultad tecnológica establece mayores exigencias a la organización y a la comunidad para mejorar significativamente, innovar y expandir los 
desarrollos tecnológicos y, por consiguiente, aumentar la capacidad instalada del proceso. Tanto la solución de los problemas operativos como las posibilidades de mejora, innovación y expansión dependen de las capacidades que haya acumulado la organización; en el primer caso de tipo operativo, y en el segundo, de tipo tecnológico.

Figura 3. Diagrama causal del microsistema de innovación

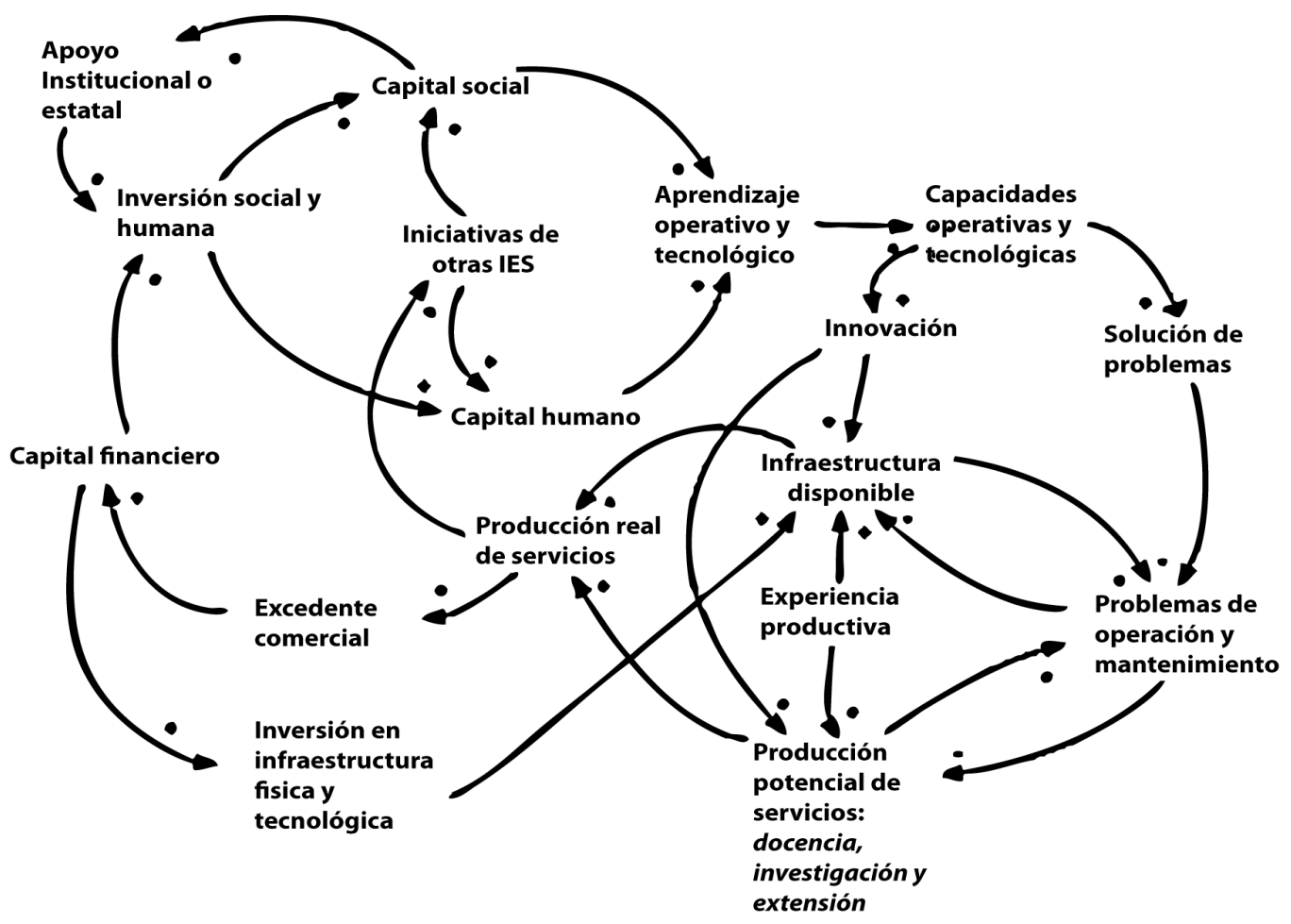

Fuente. Elaboración propia con base en Velásquez y Ceballos (2008).

- La infraestructura física y tecnológica disponible de los distintos procesos y actividades de docencia, investigación y extensión, se toma en este caso como un parámetro dado. Sin embargo, la infraestructura física y tecnológica disponible puede incrementarse como resultado de la inversión, innovación y la consecuente expansión del proceso. Esto depende del desempeño tecnológico de las unidades de investigación.

- El acceso a otros recursos como fondos económicos, información y conocimiento, depende de factores como el capital humano (en términos de capacidades operativas y tecnológicas, como ya se señaló), así como el capital social. Este último, se comparte con otras unidades de investigación, las cuales llevan en ocasiones otros procesos de innovación similares en su interior. Para medir el capital social, se puede tener en cuenta el trabajo de Giraldo-Pardo (2007), el cual integra el modelo de competitividad sistémica para las IES en Colombia, como una red social, y el cual hace mediciones de este capital a partir de los indicadores de centralidad de dicha red.

\subsubsection{Escenarios de simulación del modelo}

Siguiendo a Velásquez y Ceballos (2008), los escenarios de simulación para el caso de la introducción de una nueva tecnología, serán aquellos conformados por la dinámica entre capacidades organizacionales y desempeño tecnológico, dinámica ampliamente estudiada en la literatura de la gestión tecnológica: el escenario pesimista, en el que las capacidades son insuficientes para generar algún tipo de aprendizaje y a la vez rentabilidad; el escenario intermedio, en el que las tecnologías se sostienen con base en capacidades mínimas de operación y mantenimiento; y el escenario optimista, en el que los procesos de aprendizaje 
aseguran la acumulación de capacidades superiores, los cuales posibilitan el mejoramiento continuo y la innovación de las tecnologías. Así, los escenarios que se determinaron con base en el diagrama causal anterior, fueron:

\section{- Escenario pesimista}

No hay aprendizaje. Bajo valor del capital social y humano lo que determina obstáculos fuertes de aprendizaje que impide a la unidad de investigación llevar a cabo el proceso de aprendizaje que permita acumular las capacidades necesarias para la resolución de problemas de operación y mantenimiento, obstruye el buen funcionamiento de la tecnología hasta que esta cae en desuso sin posibilidades de recuperar la inversión que se ha hecho en el proceso de implementación y puesta en marcha.

\section{- Escenario intermedio}

Aprendizaje operativo. Los niveles de capitales social y humano no son todavía suficientes para dar el salto a la acumulación significativa de capacidades tecnológicas, pero sin embargo permiten algún grado de acumulación de capacidades operativas lo cual asegura que el desempeño alcance, con el transcurso del tiempo, los parámetros establecidos por los proveedores de la tecnología en cuestión, para satisfacer los requerimientos básicos de la unidad de investigación. Punto de equilibrio.

\section{- Escenario optimista}

Aprendizaje tecnológico. Gracias a los niveles de capitales social y humano alcanzados, hay una acumulación significativa de capacidades operativas y tecnológicas. Esto se manifiesta en el desarrollo y adaptación de nuevas tecnologías, reduciendo cada vez más el costo de la implementación y consiguiendo con ello cada vez mayor rentabilidad, gracias a la puesta en marcha de nuevos servicios como cursos on-line y videoconferencias (para dar tan sólo algunos ejemplos). Incluso, da lugar a la posibilidad de exportar tecnología o servicios relacionados hacia otras unidades de investigación, lo cual incrementa significativamente los excedentes comerciales de la institución. Ganancias.

\subsection{Modelo econométrico}

\subsubsection{Especificación del modelo}

El objetivo de este punto, consiste en analizar diferentes regímenes de ciencia y tecnología, traducidos en cambios estructurales de los Sistemas Nacionales de Innovación, considerando las fluctuaciones de la inversión y de la productividad como determinantes. Cabe anotar, que estos cambios estructurales implican procesos de aprendizaje, tanto organizacional como individual entorno a dichos sistemas. Posteriormente, se identificarán y calificarán diferentes fases o caminos de crecimiento que describan una trayectoria a largo plazo. El comportamiento oscilatorio de esa trayectoria tiene que ser atribuido a regímenes diferentes, determinados por cambios en las políticas de innovación y sus efectos sobre la estructura productiva vía cambio estructural inducido. Apoyados en la visión kaldoriana y neo-schumpeteriana introduciremos el Framework Space como instrumento de análisis de la dinámica de la trayectoria del crecimiento cognoscitivo de las Instituciones de Educación Superior.

La hipótesis de trabajo, será por lo tanto que la política de fomento a la ciencia y la tecnología, ejerce un papel importante en el proceso de crecimiento del capital (social y humano), influyendo en la trayectoria de largo plazo. La interacción entre la dinámica de corto y largo plazo generarían trayectorias de crecimiento no lineales, o sea, la trayectoria no necesariamente tiende a posiciones de equilibrio pre-determinadas (como se ve en el abordaje de la teoría convencional). Esas hipótesis iniciales nos llevan a considerar que los cambios discontinuos en la trayectoria del crecimiento que reflejan o tienen impacto sobre la estructura productiva, provocando así cambios estructurales y conformando un régimen de crecimiento. En síntesis, nuestro entendimiento sobre la dinámica del aprendizaje organizacional de una economía asocia su trayectoria de aprendizaje con cambio estructural que, a su vez, condicionará cualitativamente las trayectorias futuras de aprendizaje.

La construcción del Framework Space se inicia tomando indicadores de producción (en este caso tecnológica o bibliográfica), y de la variación de la inversión (i) en términos reales, y del recurso humano (e). Así se define: 


$$
\begin{aligned}
& \frac{d(\log v a-\log e)}{d t}=g v \\
& \frac{d(\log i-\log e)}{d t}=g i
\end{aligned}
$$

Donde $g v$ es la tasa de crecimiento del producto por investigador (una medida de la tasa de crecimiento de la productividad) y gi es la tasa de crecimiento de la inversión por investigador. Las variables $g v$ e gi proporcionan las coordenadas de la trayectoria dinámica de una determinada institución en el plano (Figura 1). Así una coordenada espacial $(g v, g i)$ es el aparato analítico del Framework Space. Alteraciones en los niveles de las coordenadas $(g v, g i)$ representan cambios en la relación dinámica de la unidad (organización) analizada, pudiendo configurar o alteraciones en la intensidad de las variables o cambios cualitativos de régimen. Cada trayectoria asociada a un comportamiento patrón de crecimiento y tratada como régimen. Así el régimen es un conjunto de trayectorias de crecimiento posibles que se comportan según la dinámica de un comportamiento patrón, esto es, sigue de un modelo canónico. Un cambio estructural coincide con un cambio de modelo de crecimiento.

La figura 3, ilustra cómo interpretar trayectorias de crecimiento y sus fases en el aparato del FS. En esa estructura son tratados seis regímenes y más un régimen especial. Este último es el espacio tangente a la línea que corta el plano de las coordenadas $(g v, g i)$ en el ángulo de $45^{\circ}$, es el llamado corredor harrodiano ${ }^{1}$, a separar el régimen VI encima de la línea, del régimen I que está debajo de esa línea. Los dos regímenes VI (acumulación) y I (innovación) están en el primer cuadrante, puesto que los regímenes están agrupados en el segundo plano siguiendo el sentido horario. En el primer cuadrante es donde ocurre el aprendizaje organizacional (o crecimiento económico), esto es, tanto la tasa de crecimiento de la productividad $(g v)$ y de la inversión por investigador ( $g i)$ crecen a tasas positivas. El régimen II (Restructuración), está en el segundo cuadrante, el $g v$ crece mientras que gi decrece (esto es, tienen valores negativos). Los demás regímenes, III, IV y V son, en la estructura analítica del FS, tratados como reflejos de los regímenes citados antes. Estos regímenes espejos tienen un comportamiento en términos de tasas de crecimiento inversamente a los regímenes VI, I y II. En el régimen III y IV las tasas de crecimiento de $g v$ y $g i$ son ambas negativas, ya que quedan en el tercer cuadrante (el cuadrante de la recesión económica). En el cuarto y último cuadrante, que coincide con el régimen $\mathrm{V}, g v$ es decreciente y gi creciente: lo que describe una situación, por ejemplo, en la cual el crecimiento de la intensidad de la inversión en innovación por investigador no se refleja en un incremento de la productividad por investigador. Ese el contrario de cualquier predicción de las variadas teorías del crecimiento.

\section{Figura 4. El Framework Space y los regímenes de} crecimiento

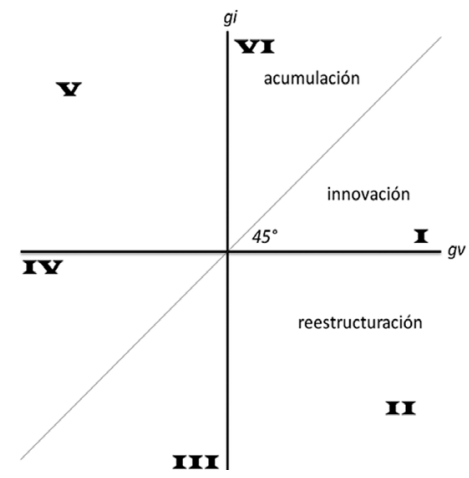

Fuente. Punzo (1995). Some complex dynamics for a multisectorial model of the economy

Una interpretación para esta argumentación es que el FS está dotado de tres patrones o regímenes de crecimiento: a) steady state; b) acumulación, enfocando los cambio en la intensidad de la inversión, y; c) la innovación, funcionalmente independiente de la acumulación de capital. Estos tres estados, pueden asociarse a los escenarios de aprendizaje antes vistos. La trayectoria del crecimiento es trazada por la secuencia formada por los pares $g v$ y $g i$, distribuidos en el plano de FS. Cada punto en el FS (definido por el par de las coordenadas) de una fase de crecimiento, estará asociado a una trayectoria de crecimiento. Para formalizar la interpretación de las trayectorias en el FS, partimos de la teoría de crecimien-to neoclásica la cual supone que la predicción del comportamiento de largo plazo de la economía, sobre ciertas condiciones, está basada en las propiedades de una función de producción, aunque en una ecuación de acumulación

\footnotetext{
${ }^{1}$ El comportamiento harrodiano es típicamente representado por la trayectoria de steady state. O sea, las razones de gv y gi son constantes. Las coordenadas $(0,0)$ se asocian al camino de crecimiento exógeno (Böhm y Punzo, 2001).
} 
de capital se puede encontrar un valor constante para la tasa de crecimiento del producto per-cápita, comúnmente conocida como la tasa de crecimiento de steady state. Esta tasa es encontrada cuando una tasa de inversión per capita requerida (necesaria para mantener una relación capitaltrabajo constante) se iguala a la inversión per capita efectiva, o sea, es la condición de equilibrio del modelo de Solow. Podemos definir que la teoría neoclásica asume una tasa de crecimiento $\mathrm{gNC}$, de steady state, dada por:

$$
g_{N C}=n+\lambda
$$

Se considera n, la tasa de crecimiento de la población, como exógena, así como la tasa de crecimiento del progreso técnico. No obstante, la tasa de crecimiento observada de las economías es diferente de la exógena. Esta diferencia es lo que se pretende explicar como auxilio del FS. Así, el FS intenta explicar la tasa endógena de crecimiento gEN dada por desvíos de la tasa observada g de la tasa de crecimiento de steady state.

$$
g_{E N}=g-(n+\lambda)
$$

El FS considerará la tasa gNC como punto de origen del diagrama de la figura 1 , como las coordenadas $(0,0)$, y de esa forma definimos aquello que es relevante para ser investigado, esto es, el análisis de la tasa de crecimiento endógena ${ }^{2}$. En ese sentido, asumimos que el comportamiento de la tasa efectiva $g$ influencia la trayectoria de largo plazo y de ese modo, asumimos que la tasa de crecimiento endógena puede ser explicada a través de dos regímenes o modelos de crecimiento: el de la acumulación de capital y el de la innovación. Esto porque tanto el ritmo de la acumulación de capital incorporado en cuanto el de la innovación en la economía influenciaran la tasa de crecimiento efectiva, consecuentemente, su trayectoria a lo largo del tiempo.
En el espacio bidimensional del FS, todas las teorías de largo plazo están presentes. El modelo de Solow (1957) está en el origen, los puntos observados fuera del origen indican crecimiento endógeno que puede ser explicado tanto por la teoría kaldoriana, régimen de acumulación de capital, en cuanto por la teoría neoschumpeteriana, régimen de innovación. En ese sentido, la elucidación de un crecimiento exógeno o endógeno puede utilizar dos clases de modelos (convencional y el kaldoriano y neoschumpeteriano, siendo los dos últimos endógenos, que estudian la dinámica de largo plazo.

Para el caso de los países estudiados, en Iberoamérica la mayoría de ellos se encontraban en un estado II (reestructuración) durante los años 1997-2002, mientras que Colombia, por ejemplo, tenía tasas de crecimiento de $g v$ y $g i$ ambas negativas, teniendo en cuenta que estaba ubicado en el tercer cuadrante (el cuadrante de la recesión económica, claro está, que en materia de investigación), al igual que Venezuela. Vale la pena mencionar que para calcular $g v$ se hizo con base en el número de publicaciones en SCI-SEARCH por cada 100 investigadores (personas físicas), mientras que $g i$, se calculó teniendo en cuenta el gasto en ciencia y tecnología por investigador (personas físicas).

Para el siguiente lustro, es decir, los años comprendidos entre 2002 y 2007, la situación cambió para muchos países, por ejemplo, Colombia pasó de estar en el cuadrante III a situarse en el eje horizontal positivo. Esto implica una mejora notable tanto en las políticas de fomento a la innovación, como también en las actividades de Investigación y Desarrollo del país. Venezuela, por su parte, continuó en una tendencia recesiva.

2 Romer (1986), Lucas (1988), Romer (1990) entre otros, buscaron endogeneizar la tasa de crecimiento al introducir conocimiento, capital humano y nuevas ideas a través de Investigación y Desarrollo en la estructura de la función de producción del modelo de Solow, esa evolución de la teoría neoclásica se conoce como "nueva teoría del crecimiento". 
Figura 5. FS y regímenes de crecimiento para los años 1997-2002

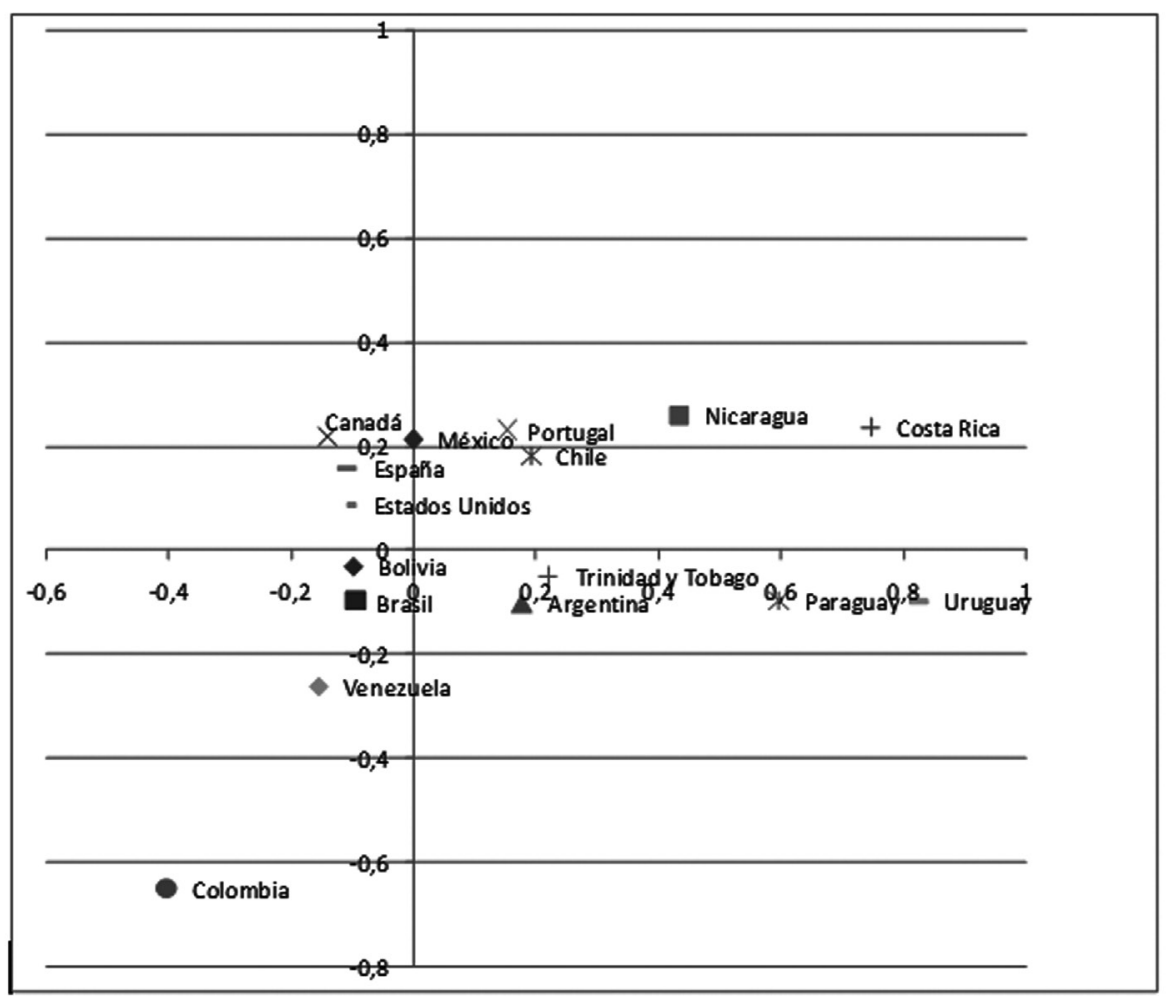

Fuente. Elaboración propia con datos de la Ricyt.

Figura 6. FS y regímenes de crecimiento para los años 2002-2007

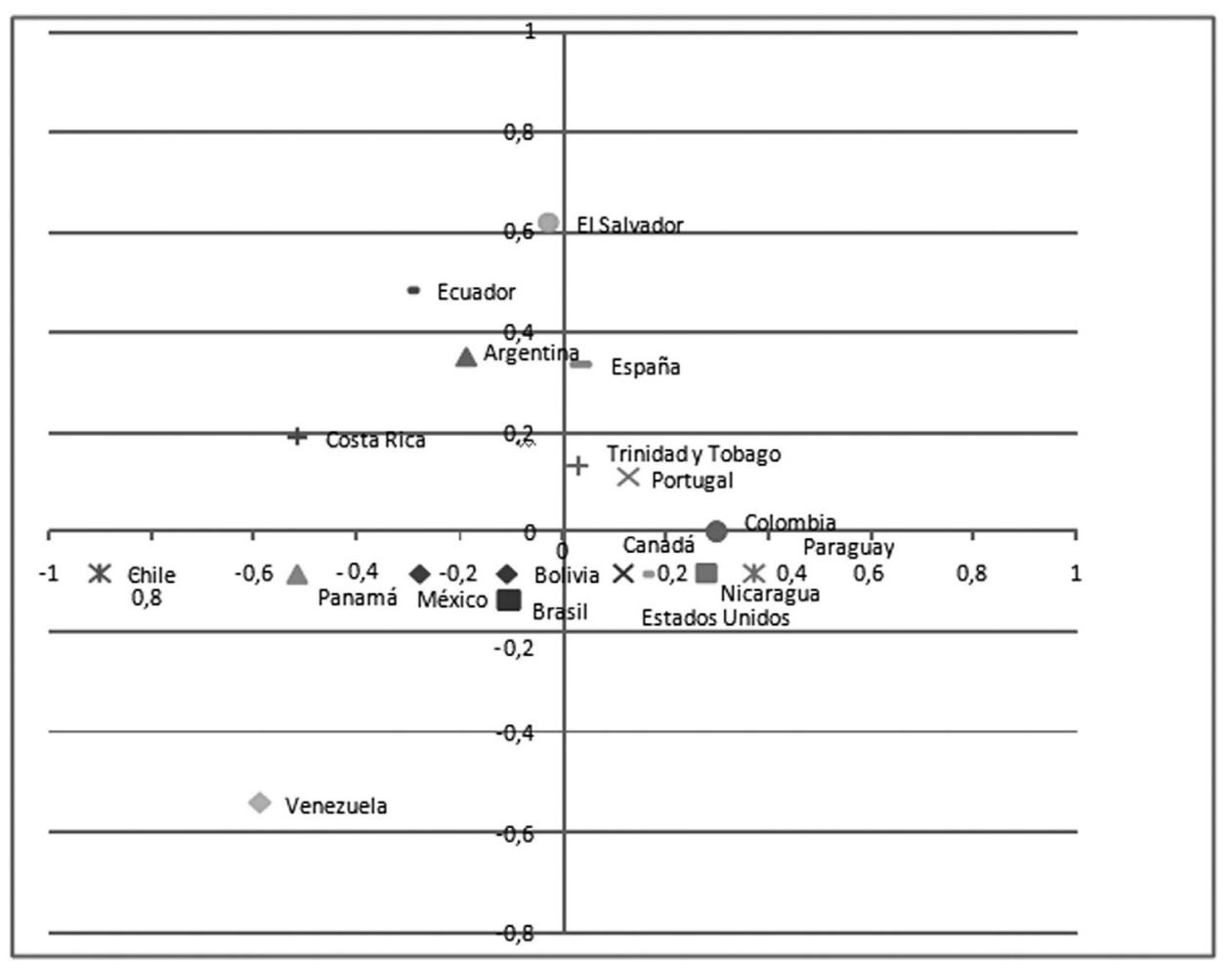

Fuente. Elaboración propia con datos de la Ricyt. 
En el caso de Estados Unidos, se dio un efecto de recuperación de la inversión, ya que mientras se invirtió entre los años 1997-2002 con decrementos en la tasa de productividad. Esta tasa se volvió creciente entre los años 2002-2007, posiblemente como consecuencia de las políticas de fomento precedentes.

\subsubsection{Metodología para evaluar la sustentabilidad de los proyectos de inversión en innovación para la IES}

Teniendo en cuenta que la metodología anterior, fue expuesta para analizar diferentes regímenes de ciencia y tecnología, ahora debemos evaluar los distintos componentes de la sustentabilidad, tales como la sostenibilidad y la pertinencia (ecuación 1 de la figura 1) de las políticas de innovación.

Para el caso de la sostenibilidad, esta se obtiene a partir de una relación de dos variables básicas que la componen, esto es, el producto (iniciativa a la estructura) y la inversión por investigador (consideración), de cuadro con la ecuación 2 de la figura 1 . Con el fin de evaluar este componente en los diferentes países, se hicieron figuras de dispersión que relacionaban la consideración ${ }^{3}$ (gasto por investigador en miles de dólares ppa) en el eje horizontal, con la iniciativa a la estructura ${ }^{4}$ (promedio de las variables coeficiente de invención y publicaciones en science citation, ajustadas ${ }^{5}$ ), en el eje vertical. Los resultados se encuentran en las figuras 1.1, 1.2 y 1.3 del anexo. De acuerdo con esto, los países con políticas más sostenibles de investigación y desarrollo, para el año 1997 fueron Argentina, Venezuela y EE.UU (véase gráfico 1.1). Los demás países, a excepción de Canadá, España y Brasil, tenían niveles muy bajos de sostenibilidad

De la misma manera, se pueden establecer relaciones similares para las demás ecuaciones de la figura 1.

Para el caso de la ecuación 3, la responsabilidad social es el resultado de la conjunción de los conceptos de consideración y pertinencia. Para el caso de la consideración, se toma de nuevo el gasto por investigador (en miles de dólares ppa), mientras que la pertinencia, será el promedio (aritmético) de tres variables ajustadas ${ }^{6}: 1$. Gasto en CyT por Tipo de Actividad 2. Gasto en CyT por Sector de Financiamiento. 3. Gasto en CyT por Sector de Ejecución. 4. Gasto en CyT por Objetivo Socioeconómico 5. Créditos Presupuestarios públicos en $\mathrm{I}+\mathrm{D}$ por Objetivo Socioeconómico. 6. Gasto en CyT por disciplina científica $^{7}$. Los resultados para la responsabilidad social, pueden verse en los gráficos 2.1, 2.2 y 2.3 del Anexo. En términos generales, los niveles de pertinencia de las políticas de CyT

3 La consideración, entendida como la acción de "tener en cuenta", implica la inclusión de actores o ideas dentro de algo más amplio. En este caso, está enmarcada precisamente en la inclusión de los actores internos a los procesos de investigación e innovación, como parte activa de cada uno de estos procesos en relación a la responsabilidad que se tiene con cada uno de ellos. La medida más factible encontrada en este momento para estimar esta variable, podría ser el gasto por investigador, teniendo en cuenta, que a medida que aumenta este, los investigadores tienen más recursos, no sólo para llevar a cabo sus labores, sino también para cualificarse más. Este también podría verse como un indicador de "nivel de vida", como lo es el producto per cápita, solamente que enmarcado en el ámbito de la ciencia y la tecnología.

4 Como ya se explicó en el marco teórico, la iniciativa a la estructura, relacionada directamente con la consecución de resultados de un proceso.

5 El ajuste consiste en aplicar el siguiente factor, el cual permite la estandarización de las unidades, pero a la vez, ayuda a visualizar el orden de los países analizados: $x-m / M-m$

Donde $\mathrm{m}$ es el mínimo, $\mathbf{M}$ es el máximo y x el valor de la variable para el país estudiado.

6 Para el caso de la pertinencia, se tendrá en cuenta la variabilidad de cada una de las dos variables antes mencionadas, de manera que a menor acumulación en unos pocos rubros para cada una de estas variables, mayor será la pertinencia, satisfaciendo a un mayor número de actores. Para cada una de estas variables (tomando su varianza), el factor de ajuste será en siguiente: $\quad M-x / M-m$

Donde $\mathrm{m}$ es el mínimo, $\mathrm{M}$ es el máximo y x el valor de la variable para el país estudiado.

7 Para seleccionar estas variables, se tuvo en cuenta que la pertinencia, se define como "la dimensión que comprueba que los objetivos propuestos por las instituciones corresponden a los requeridos desde una perspectiva externa” Latapí (1995). Por otra parte, González-Guitián y MolinaPiñeiro (2009), definen los siguientes indicadores de pertinencia en los ámbitos de la Ciencia y la Tecnología en Cuba:

- Porcentaje de proyectos vinculados a proyectos nacionales, ramales, territoriales, empresariales y universitarios en ciencia y tecnología.

- Proyectos en planes de generalización ramales y provinciales.

- Financiamiento de los proyectos de investigación en peso cubano convertible (CUC).

González Guitián y Molina Piñeiro: La evaluación de la ciencia: revisión 
se mantienen altos y estables entre los años 1997 y 2007, mientras que por otra parte, se incrementa el componente de consideración a lo largo de este periodo, lo que muestra una evolución en términos de responsabilidad social para la mayoría de países.

Ahora bien, para el caso de la ecuación 1, en la cual se define la sustentabilidad en términos de la sostenibilidad y la pertinencia. Se toman exactamente los mismos indicadores que se desarrollaron para cada una de ellas en las ecuaciones 2 y 3 . Los resultados, se encuentran en las figuras $3.1,3.2$ y $3.3 \mathrm{del}$ anexo. A lo largo del periodo estudiado, se conservan casi los mismos niveles de pertinencia para todos los países, pero hay una evolución en términos de sostenibilidad.
Por otra parte, para robustecer las conclusiones obtenidas, también se seguirá el mismo procedimiento con la ecuación 4 de la figura 4.1, en la cual se expresa la sustentabilidad en términos de la responsabilidad social y la iniciativa a la estructura. Los resultados, se encuentran en las figuras 4.1, 4.2 y 4.3 del anexo. La mayoría de países conservan unos niveles medios de responsabilidad social a lo largo de todo el periodo, a excepción de algunos países como Nicaragua y Honduras, que poseen unos niveles de "responsabilidad social" extremadamente bajos o Venezuela, que siempre ostentó un nivel elevado de "responsabilidad social". Para cada uno de los tres años estudiados, el único país con alto grado de iniciativa a la estructura fue Estados Unidos, y por lo tanto, el más sustentable desde esta perspectiva, junto con Venezuela.

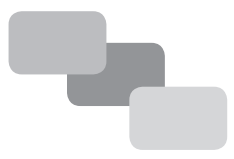

\section{CONCLUSIONES}

partir de una visión multidimensional del problema de la sustentabilidad en el ámbito de la ciencia, la tecnología y la innovación, es posible hacer diagnósticos detallados sobre la eficacia y la eficiencia de las políticas de fomento, cuando se tienen múltiples objetivos y stackeholders. Además, esta visión plural es importante teniendo en cuenta la complejidad mencionada desde el comienzo de este artículo, implícita en los procesos de innovación, si bien, esta muchas veces no es el resultado de un proceso planeado sino algo espontáneo. No obstante, todos los gobiernos a nivel mundial están cada vez más interesados en desarrollar estrategias que promuevan el desarrollo de la ciencia y la tecnología como parte de sus programas de desarrollo económico.

Otro aspecto relevante, que está implícito en la metodología diseñada, es el carácter dual de la innovación, la cual es un terreno fértil para el estudio de la sustentabilidad, a diferencia de otros ámbitos de decisión pública o privada, debido a que son equiparables en términos de complejidad. Este carácter dual, es compatible igualmente con el carácter dual de las dos definiciones de sustentabilidad desarrolladas en este trabajo, lo cual nos remite a una evolución de los aspectos económicos y organizacionales en paralelo con una comprensión mayor de los mismos, los cuales se vislumbran en la jerga y en las teorías actuales sobre crecimiento y desarrollo.

Esa complejidad, y la conciencia de ella que es el fundamento del paradigma sistémico, el cual se muestra nuevamente como una herramienta indispensable para la comprensión de los fenómenos actuales, comprende no sólo los fenómenos de recursividad y recurrencia implícitos en la construcción de los conceptos (en este caso el de sustentabilidad y el de innovación), sino también en la relación dinámica de los mismos, que conforman lo que conocemos en la vida real como tendencias o evoluciones.

Finalmente, se espera que los diagnósticos suministrados a lo largo de este trabajo de investigación, se conviertan algún día en un insumo para la toma de decisiones estratégicas en el ámbito de la ciencia y la tecnología, así como para la formulación de políticas de fomento a la innovación. 


\section{ANEXDS}

Figura 1.1. AÑO 1997: sostenibilidad = consideración $($ eje $x)+$ iniciativa a la estructura (eje y)

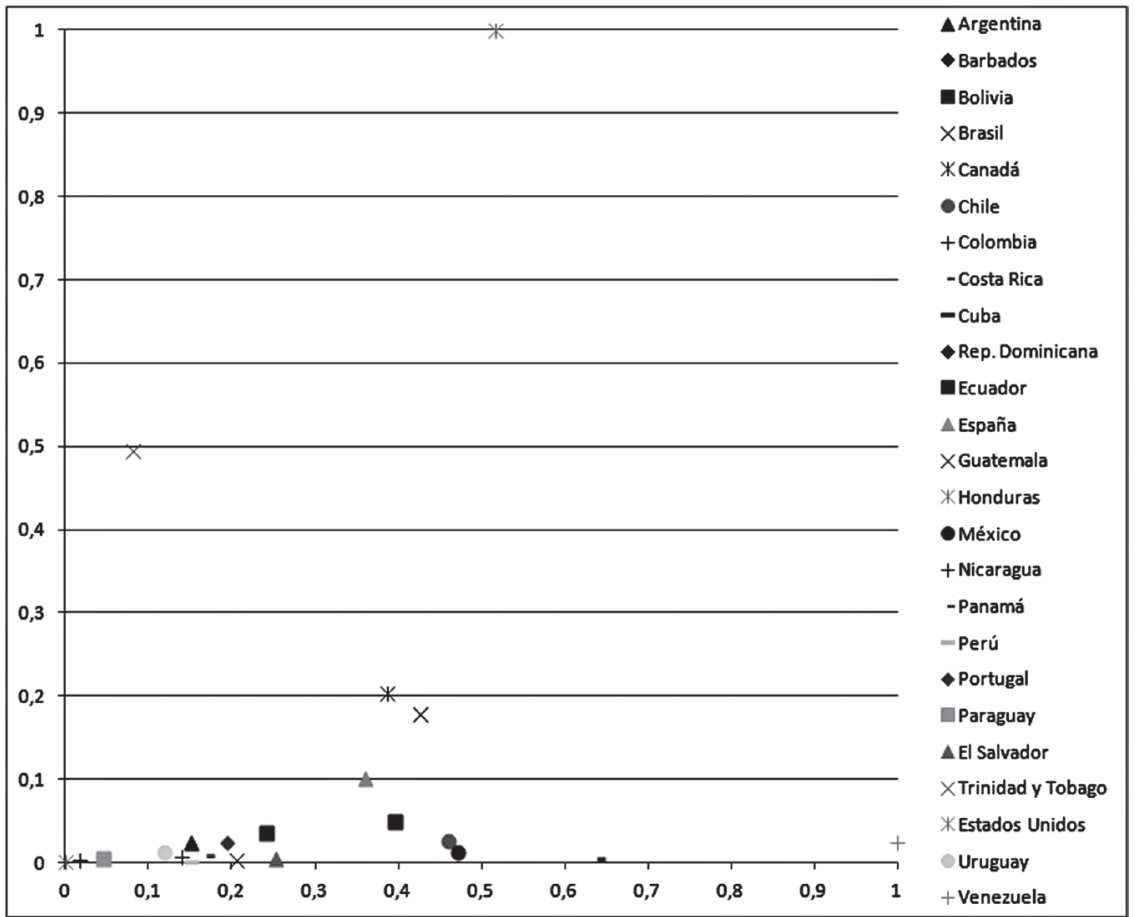

Fuente. Elaboración propia con datos de la Ricyt.

Figura 1.2. año 2002: sostenibilidad $=$ consideración $($ eje $x)+$ iniciativa a la estructura (eje y)

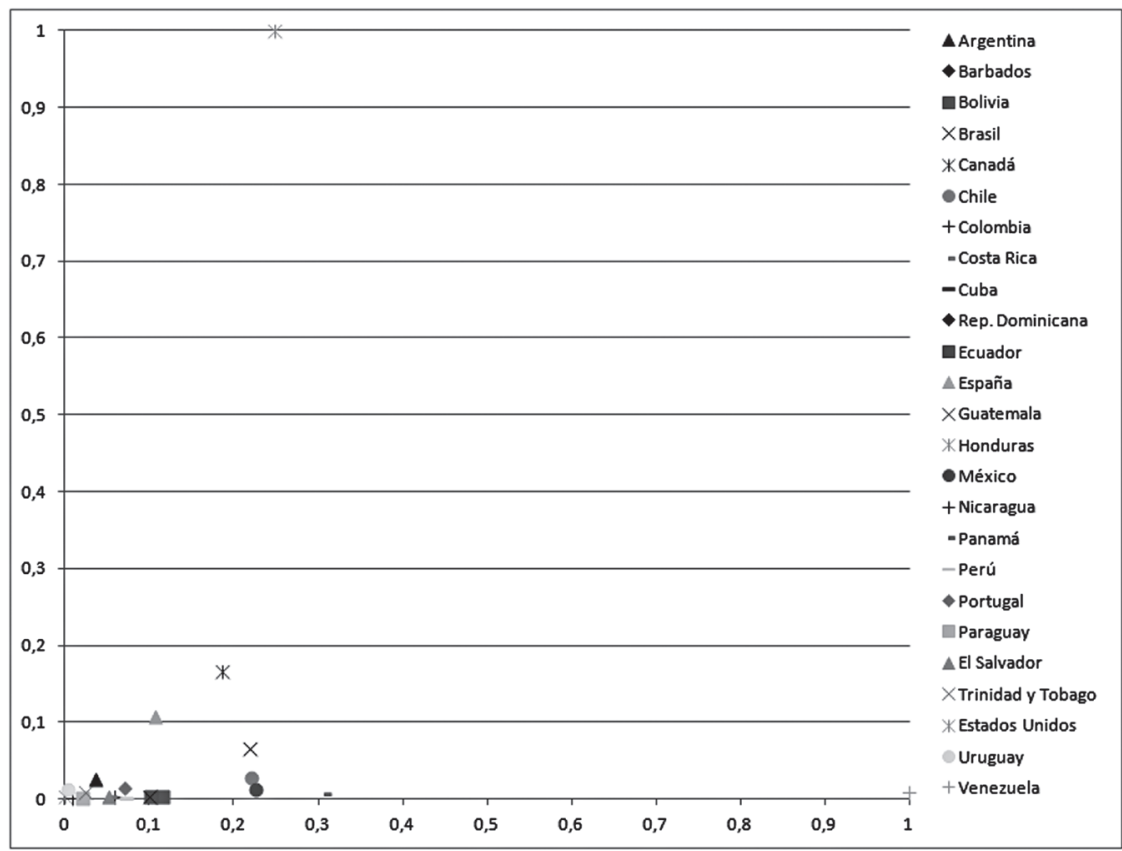

Fuente. Elaboración propia con datos de la Ricyt 
Figura 1.3. año 2007:sostenibilidad $=$ consideración (eje $x)+$ iniciativa a la estructura (eje y)

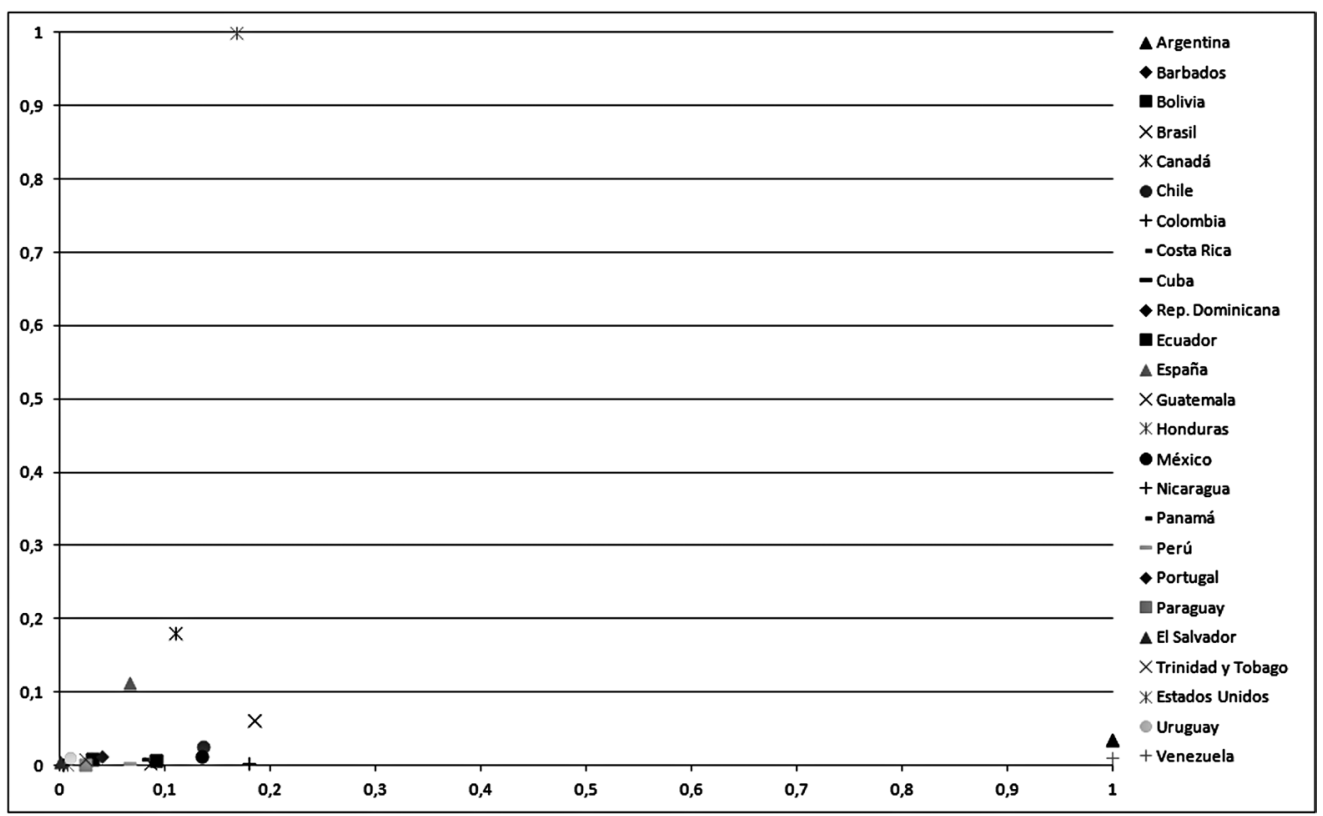

Fuente. Elaboración propia con datos de la Ricyt

Figura 2.1. año 1997: Responsabilidad social = pertinencia $($ eje $x)+$ consideración $($ eje y)

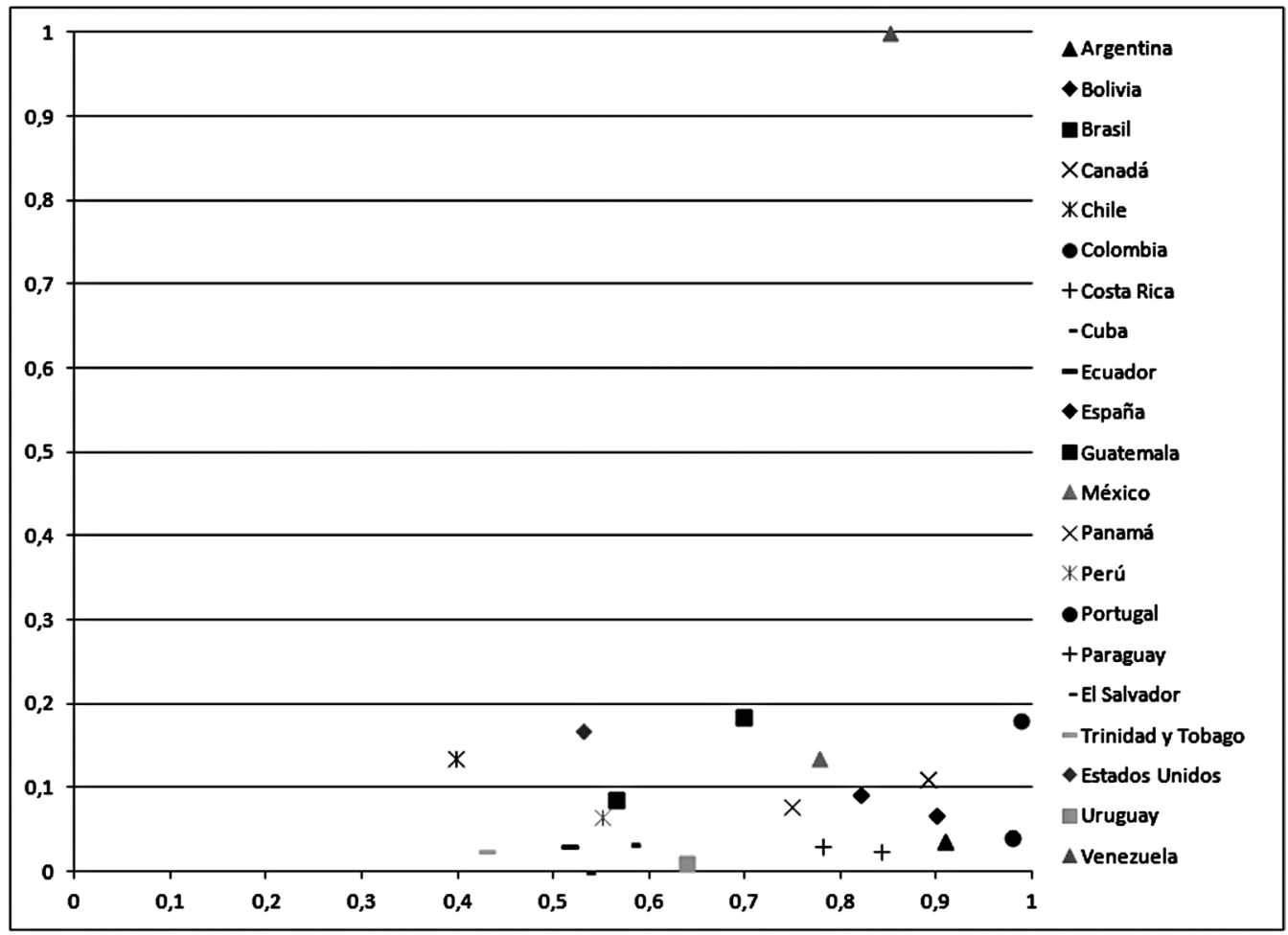

Fuente. Elaboración propia con datos de la Ricyt 
Figura 2.2 año 2002: responsabilidad social = pertinencia $($ eje x)+ consideración (eje y)

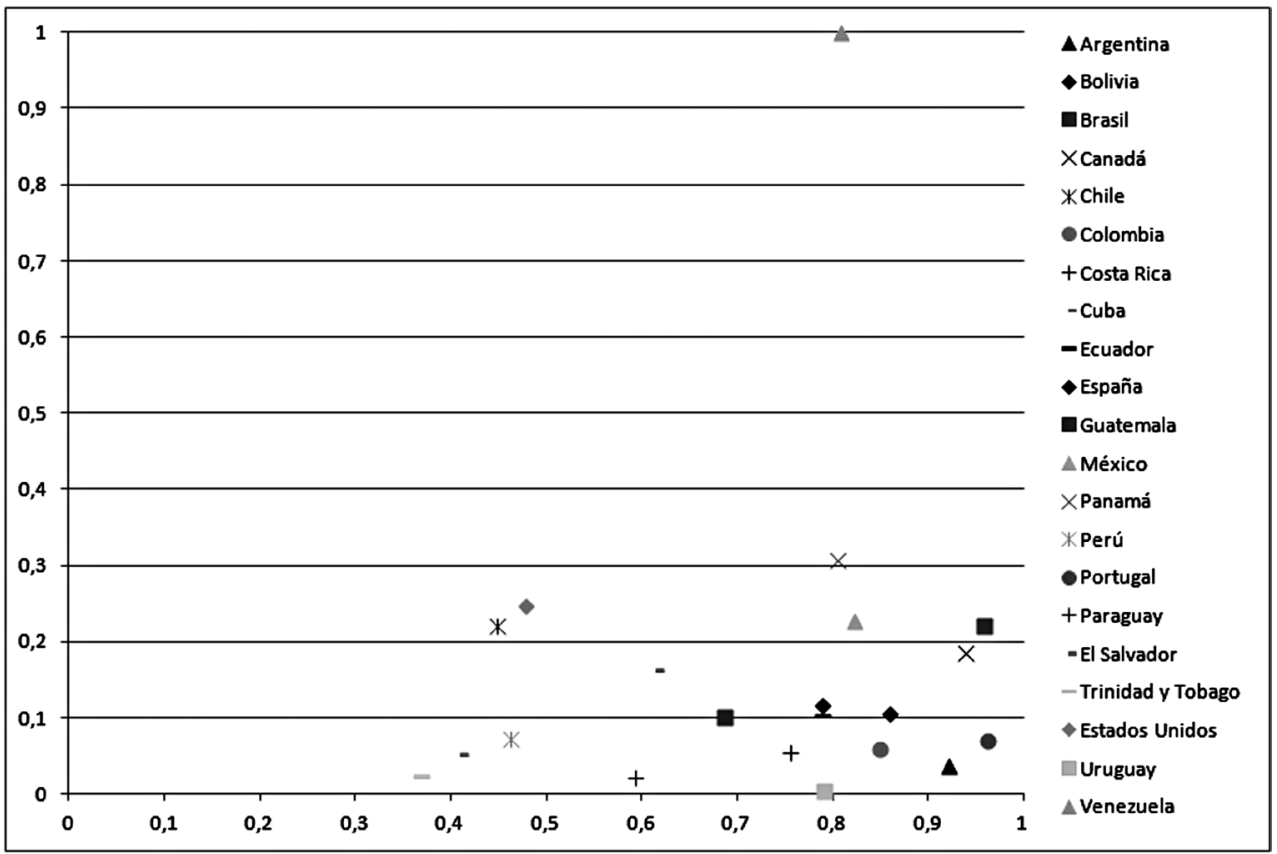

Fuente. Elaboración propia con datos de la Ricyt

Figura 2.3 año 2007: responsabilidad social = pertinencia $($ eje $x)+$ consideración $($ eje y)

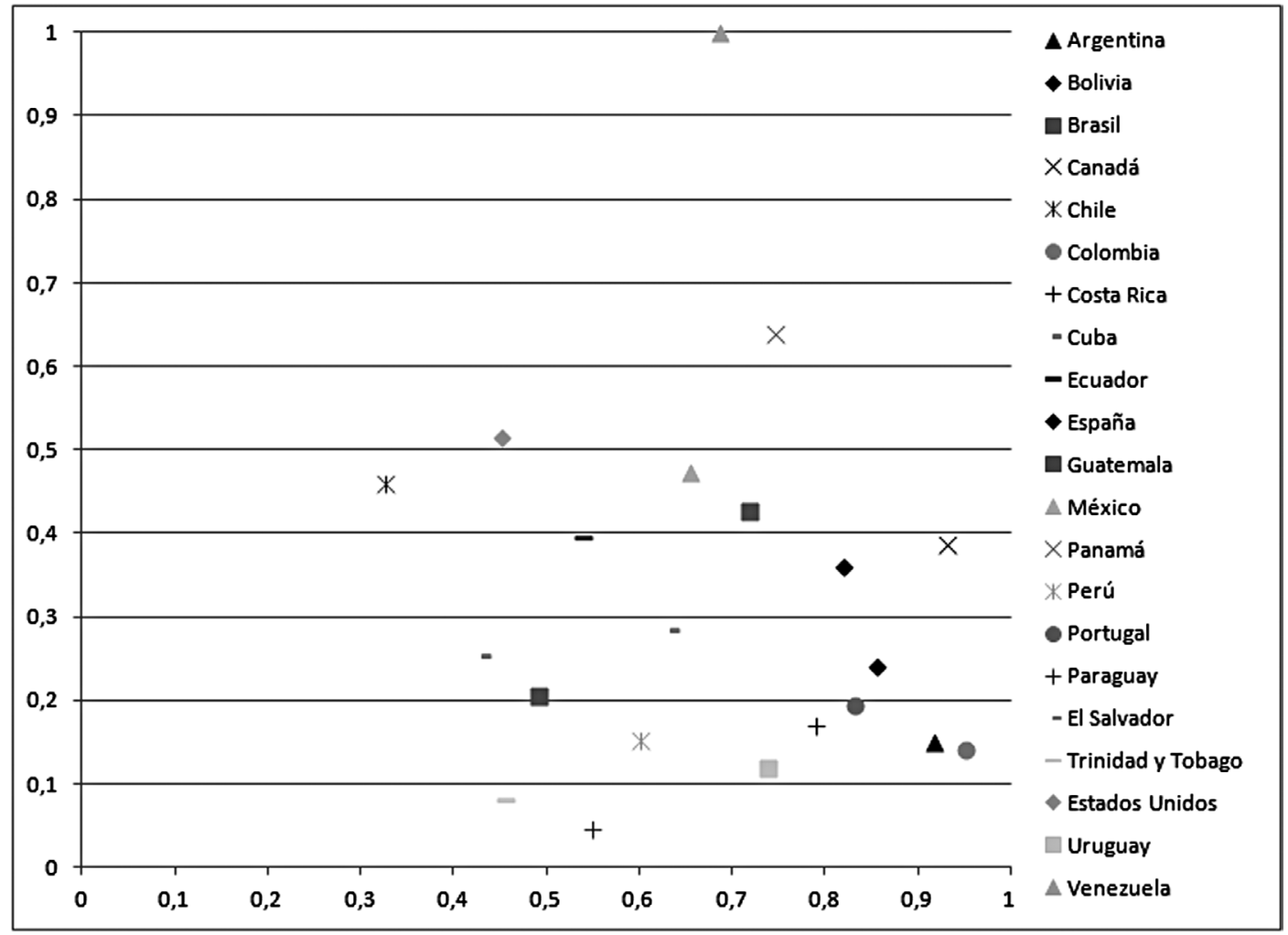

Fuente. Elaboración propia con datos de la Ricyt 
Figura 3.1 año 1997: sustentabilidad $=$ sostenibilidad $($ eje $x)+$ pertinencia $($ eje y)

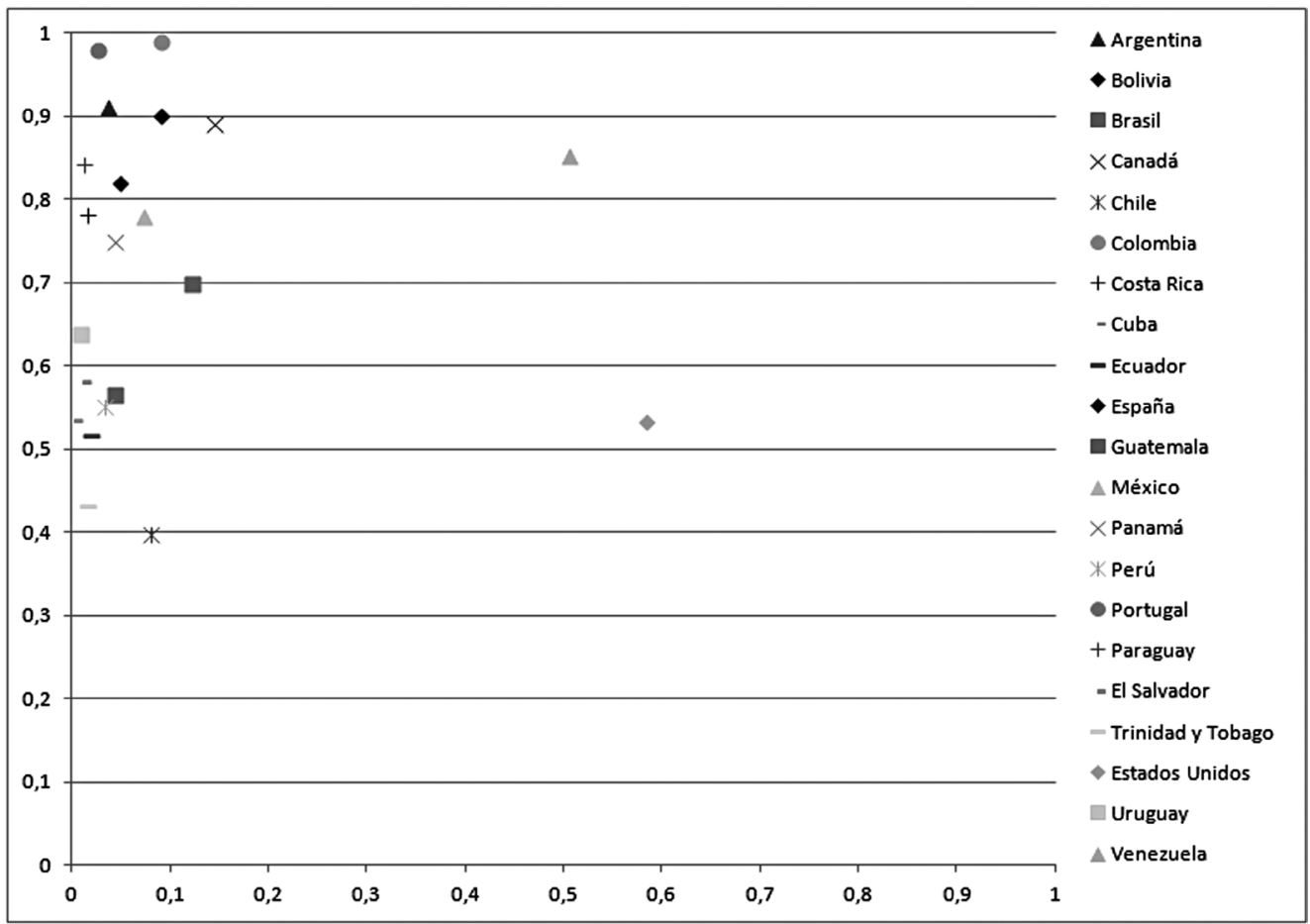

Fuente. Elaboración propia con datos de la Ricyt

Figura 3.2 año 2002: sustentabilidad $=$ sostenibilidad $($ eje $x)+$ pertinencia $($ eje $y)$

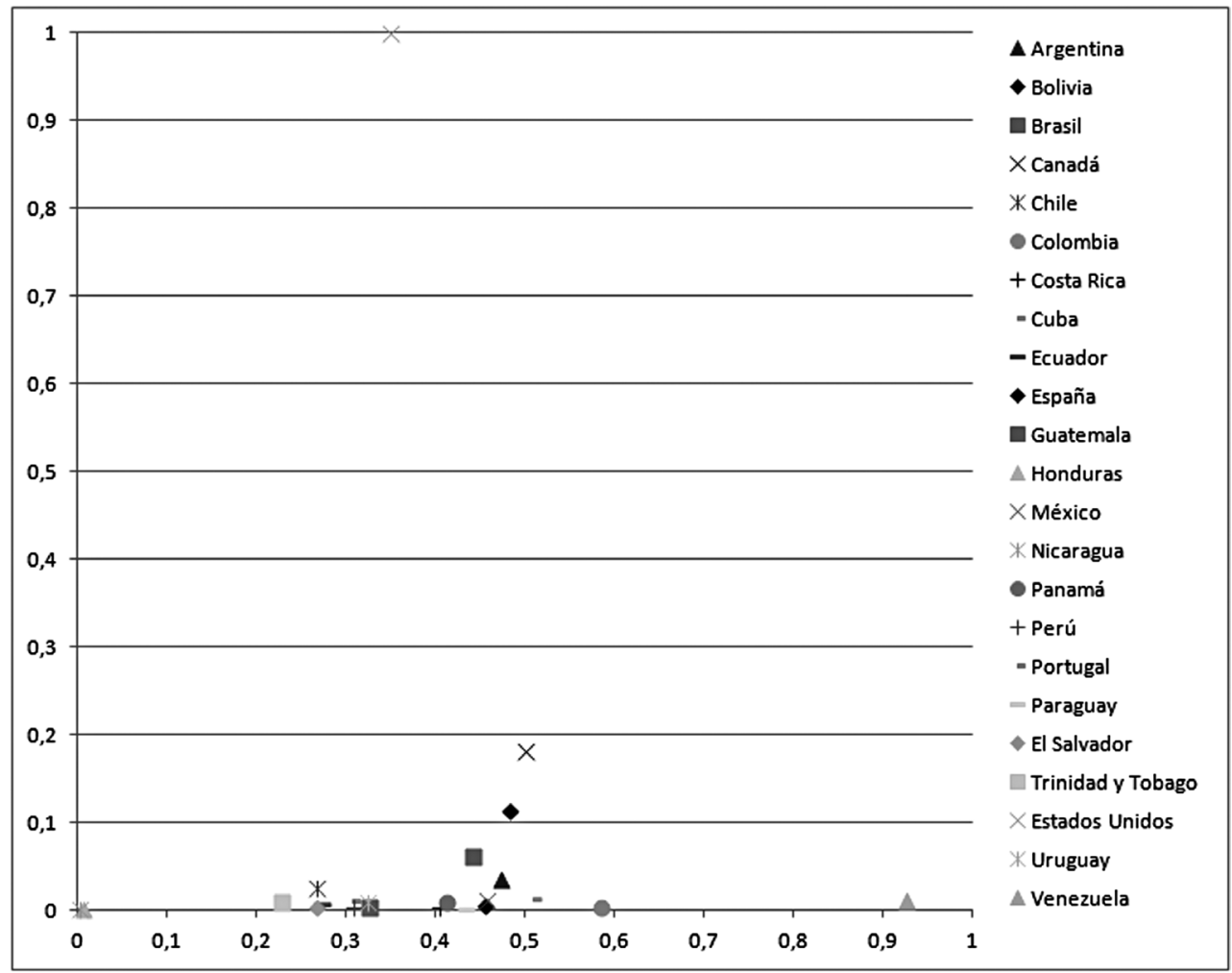

Fuente. Elaboración propia con datos de la Ricyt 
Figura 3.3 AÑO 2007: sustentabilidad = sostenibilidad $($ eje $x)+$ pertinencia $($ eje y)

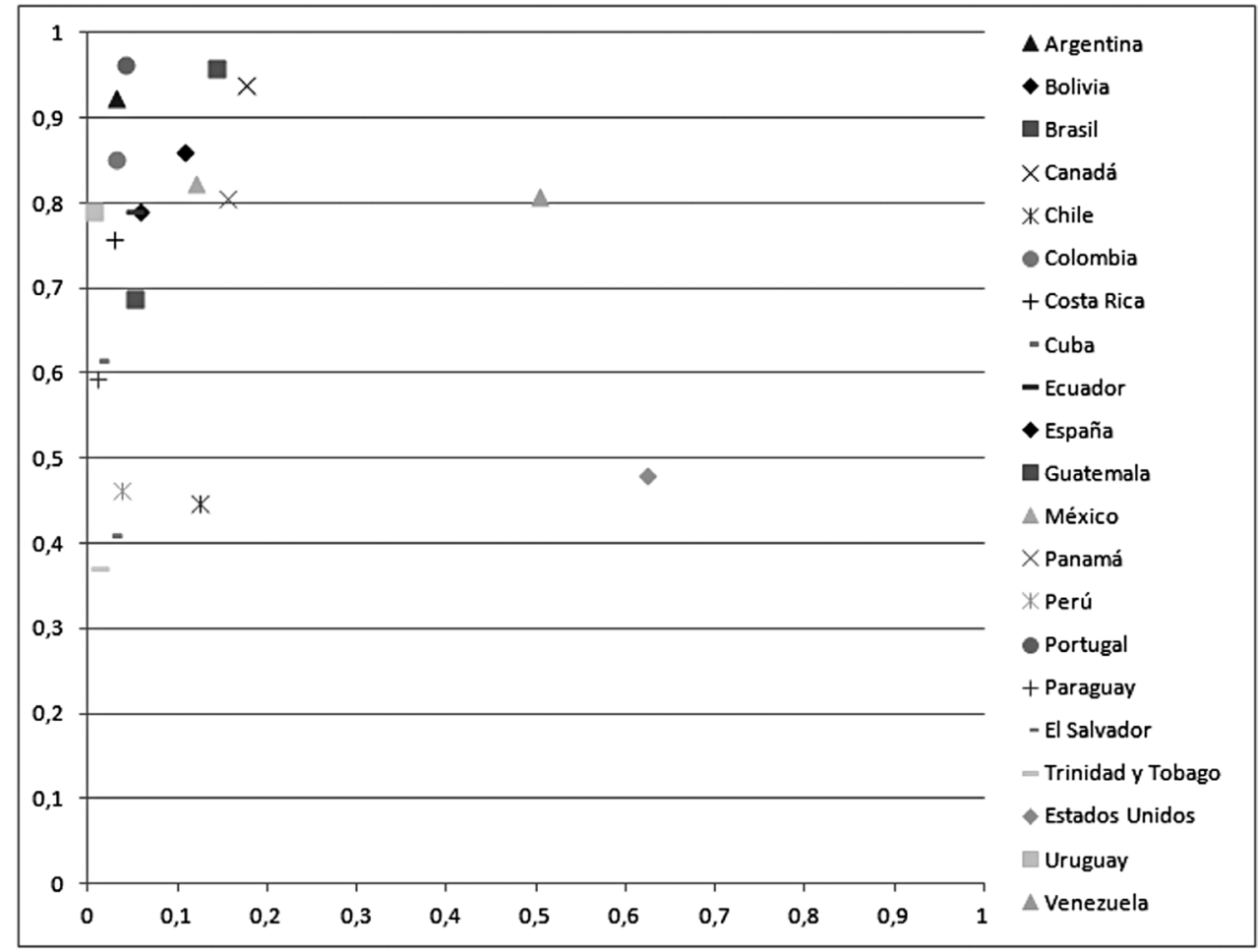

Fuente. Elaboración propia con datos de la Ricyt

Figura 4.1 año 1997: sustentabilidad = responsabilidad social (eje $x)+$ iniciativa a la estructura (eje y)

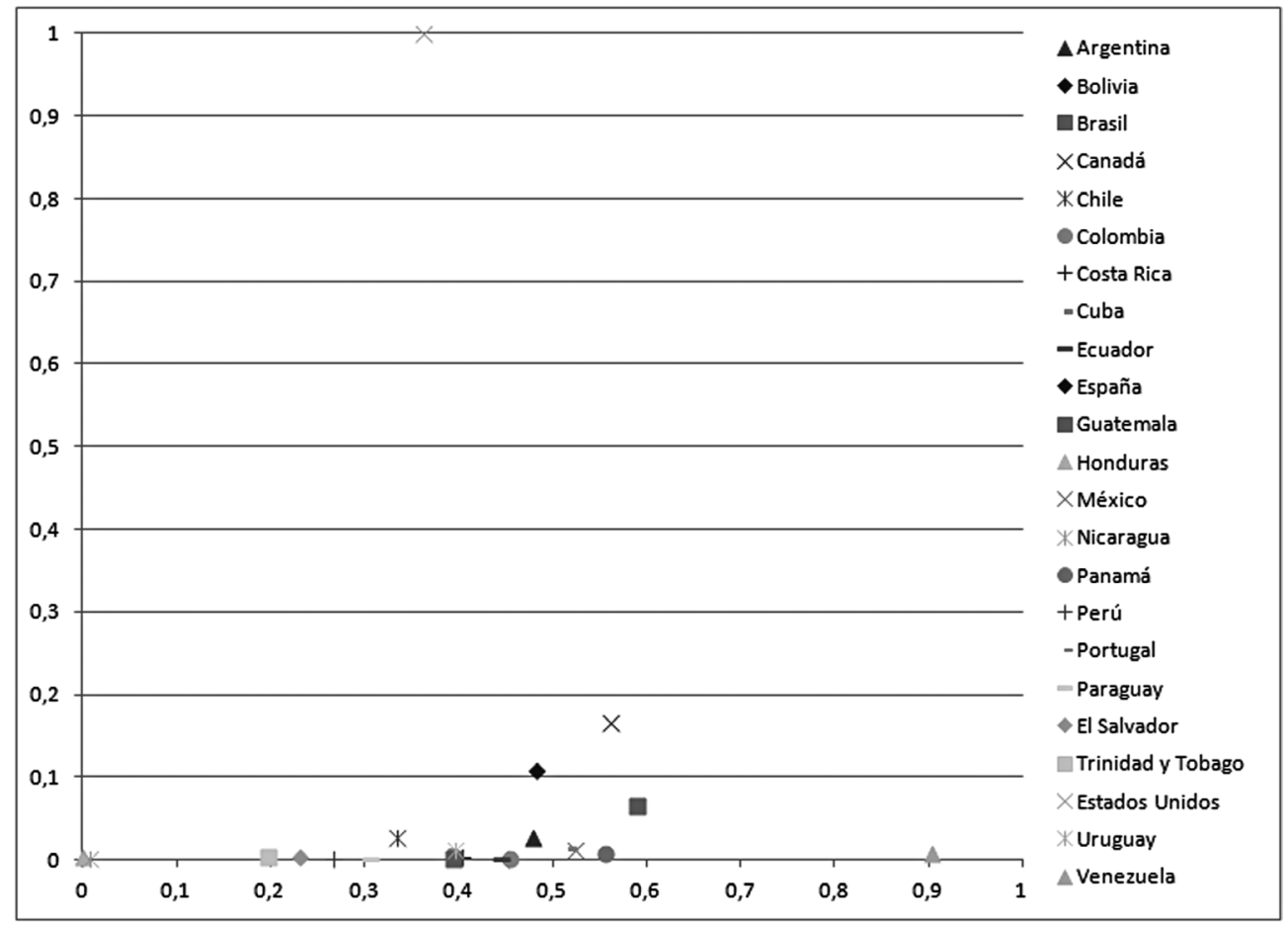

Fuente. Elaboración propia con datos de la Ricyt 
Figura 4.2 año 2002: sustentabilidad $=$ responsabilidad social (eje $x)+$ iniciativa a la estructura (eje $y)$

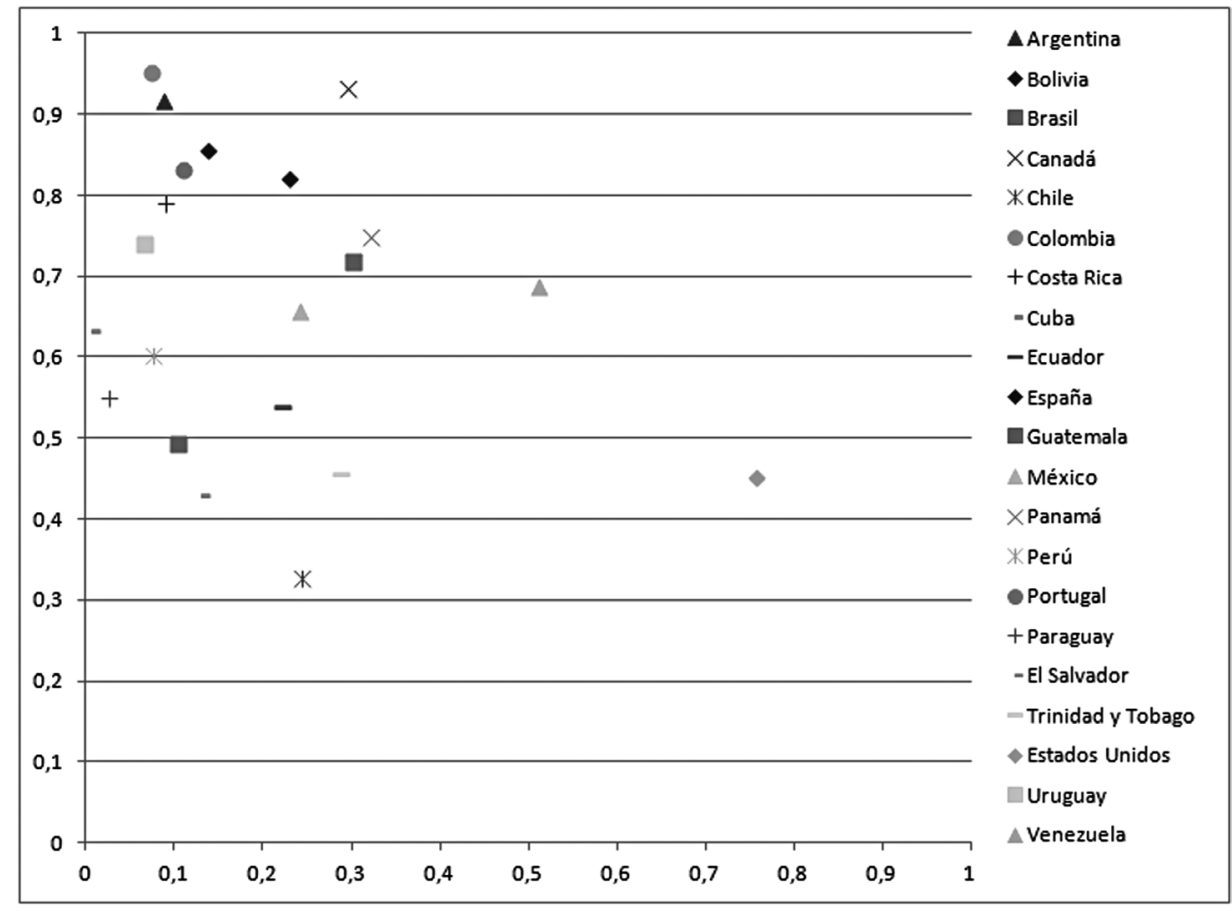

Fuente. Elaboración propia con datos de la Ricyt

Figura 4.3 año 2007: sustentabilidad $=$ responsabilidad social $($ eje $x)+$ iniciativa a la estructura (eje y)

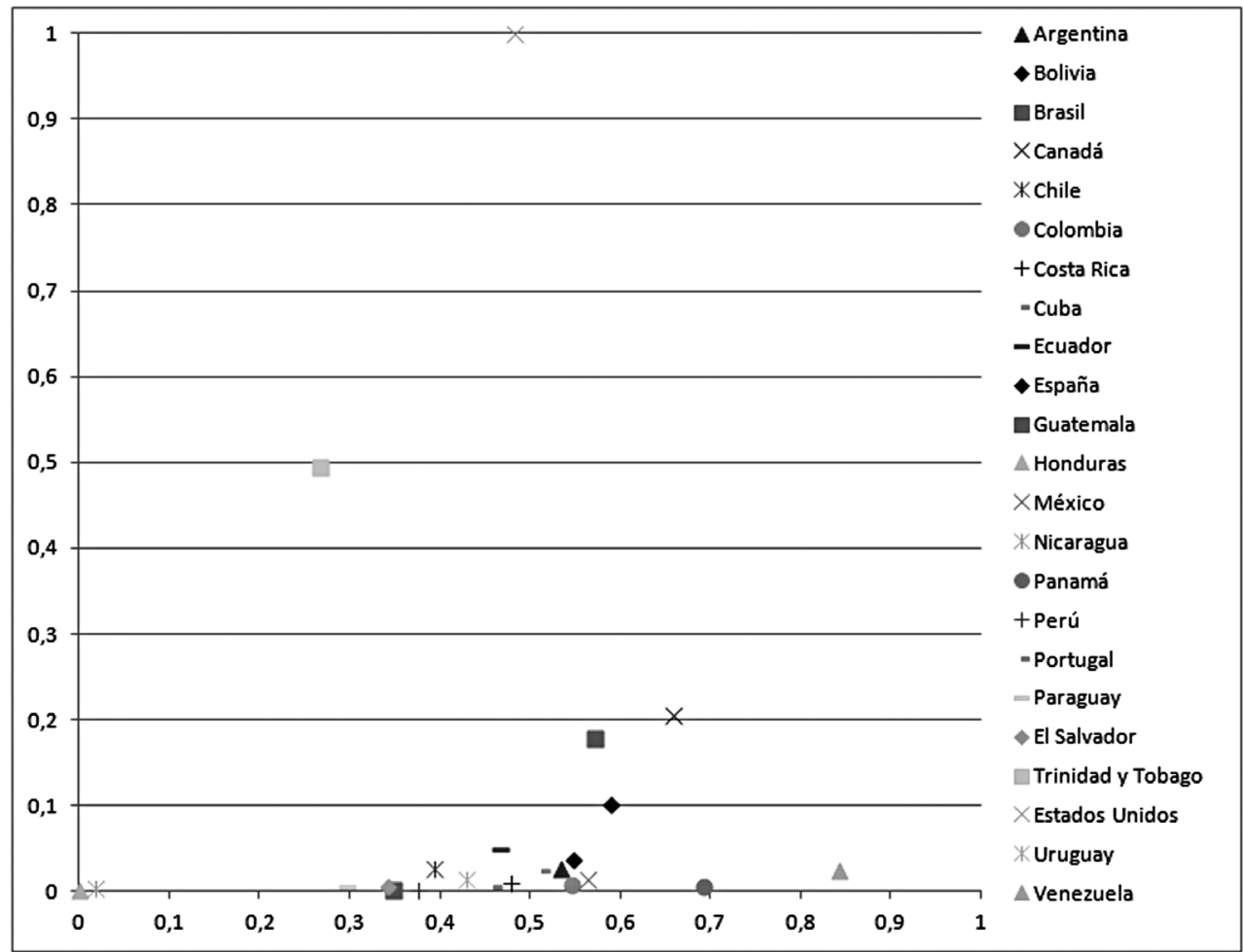

Fuente. Elaboración propia con datos de la Ricyt 


\section{Б. REFERENCIAS BIBLIOGRÁFILAS.}

Andrade, H; Parra, J; Pineda, E. (2003). Dinámica de Sistemas y Econometría: en la búsqueda de posibilidades o imposibilidades de reconocimiento a partir de la reflexión de un experiencia en Economía: en II Encuentro Colombiano de Dinámica de Sistemas.

Arellano C., W. (2003). Educación Superior y Pertinencia Social, Plan estratégico de Cartagena siglo XXI, Marzo de 2003, Cartagena-Colombia.

Argyris y Schön (1978). Organizational Learning: A Theory of Action Perspective. Addison-Wesley.

Bell, M. (1984). Learning and the accumulation of industrial technological capacity in developing countries. In: M. Fransman and K. King (Eds.), Technological capability in the third world (pp. 187-209). London: Macmillan.

Chanal (2002). How to design innovative organizations: a focus on practice and conversations. EGOS conference.

Cooke, Urange, Extebarria (1997). Regional innovation systems: institutional and organizational dimensions. Research Policy 4 y 5.

Freeman, C. (1974). La teoría económica de la innovación industrial. Editorial Alianza Universidad.

Freeman, C. y Pérez, C. (1988). Structural crisis of adjustment, business cycles and investment behaviour. En: G. Dosi et al. (Ed.), Technical change and economic theory (pp. 38-66). London: Pinter Publisher.

Giddens, A. (1998). The constitution of society, Polity Press. Cambridge.

Giraldo-Pardo, T. (2008) Innovación Tecnológica en la Academia: Una mirada desde la perspectiva sistémica y sociotécnica. Memoire D.U. en Sciences de Gestion Université de Rouen, Bogotá-Colombia.

Henderson, R y Cockburn, I. (1994). Measuring competente: Exploring Firm-Effects in Pharmaceutical Research. Strategic Management Journal. 15 (Winter Special Issue). 63-84.

Kaldor, N. (1957). A Model of Economic Growth, Economic Journal, 1957.

Kaldor, N. y Mirrlees, J.A.(1961). A New Model of Economic Growth, Review of Economic Studies, vol. 29, 1961-1962.

Latapí, P. (1995). El Plan Nacional de Educación y el discreto robo del tiempo. Proceso, 972. Junio de 1995. México D.F.

Letelier, M. (2002). Sustentabilidad, Sostenibilidad y Pertinencia en la Educación Superior. CICES - Universidad de Santiago de Chile. Santiago de Chile.

López-Ricalde, López-Hernández y Ancona (2005). Desarrollo sustentable o sostenible: una definición conceptual. Revista horizonte sanitario. No. 2, Vol. 4. 
Malagón, L. (2003). La pertinencia en la educación superior. Elementos para su comprensión. En Revista de la Educación Superior. Vol. 3, 127. Julio-septiembre de 2003.

Nonaka, I. Toyama, R. Y Nagata, A. (2000). A firm as a knowledge-creating entity: A new perspective on the theory of the firm: Industrial and Corporate Change; ABI/INFORM

Robbins, S. (2004). Comportamiento organizacional: Conceptos, controversias y aplicaciones. Ed. Pearson. México. Rogers (2003). Diffusion of Innovations. Free Press. Nueva York.

Schumpeter, J. A. (1934). The theory of economic development. Harvard University Press, Boston.

Schumpeter, J. A. (1947). The Creative Response in Economic History. Journal of Economic History. (7). 149-159.

Schumpeter, J. A. (1950). Capitalism, Socialism and Democracy. Nueva York: 3ar ed. Harper.

Tünnermann (2006). Pertinencia y calidad de la educación superior. Lección inaugural. Guatemala, 31 de enero, 2006.

Velásquez, J. y Ceballos, Y. (2008) Estudio de un proceso de innovación utilizando la dinámica de sistemas. Cuadernos de Administración 2008, 21 (enero-junio). Universidad Javeriana. Bogotá.

Vercelli (1998) Operational measures of sustainable development and the freedom of future generations. En: G. Chichilnisky, G. Heal and A. Vercelli (Editors), Sustainability: Dynamics and Uncertainty, Kluwer, Dordrecht.

Winter, S. (2000). The satisficing principle in capability learning. Strategic Management Journal, 21, 981-996. 